\title{
GLOBAL WELL-POSEDNESS IN THE CRITICAL BESOV SPACES FOR THE INCOMPRESSIBLE OLDROYD-B MODEL WITHOUT DAMPING MECHANISM
}

\author{
QIONGLEI CHEN AND XIAONAN HAO
}

\begin{abstract}
We prove the global well-posedness in the critical Besov spaces for the incompressible Oldroyd-B model without damping mechanism on the stress tensor in $\mathbb{R}^{d}$ for the small initial data. Our proof is based on the observation that the behaviors of Green's matrix to the system of $\left(u,(-\Delta)^{-\frac{1}{2}} \mathbb{P} \nabla \cdot \tau\right)$ as well as the effects of $\tau$ change from the low frequencies to the high frequencies and the construction of the appropriate energies in different frequencies.
\end{abstract}

\section{INTRODUCTION}

We are concerned with the incompressible Oldroyd-B model of the non-Newtonian fluid in $\mathbb{R}^{+} \times \mathbb{R}^{d}$

$$
\left\{\begin{array}{l}
u_{t}+u \cdot \nabla u-\nu \Delta u+\nabla p=\mu_{1} \nabla \cdot \tau, \\
\tau_{t}+u \cdot \nabla \tau+a \tau+Q(\tau, \nabla u)=\mu_{2} D(u), \\
\nabla \cdot u=0, \\
u(0, x)=u_{0}(x), \quad \tau(0, x)=\tau_{0}(x) .
\end{array}\right.
$$

Here $u(t, x)$ stands for the velocity fluid and $\tau(t, x)$ is the non-Newtonian part of stress tensor $\left(\tau\right.$ is a $d \times d$ symmetric matrix and $\left.[\nabla \cdot \tau]^{i}=\sum_{j} \partial_{j} \tau^{i, j}\right)$. The pressure $p$ is a scalar and coefficients $\nu, a, \mu_{1}, \mu_{2}$ are assumed to be non-negative constants. The bilinear term $Q$ has the following form

$$
Q(\tau, \nabla u)=\tau W(u)-W(u) \tau-b(D(u) \tau+\tau D(u)),
$$

where $b \in[-1,1]$, and $D(u)=\frac{1}{2}\left(\nabla u+(\nabla u)^{\top}\right), W(u)=\frac{1}{2}\left(\nabla u-(\nabla u)^{\top}\right)$ are the deformation tensor and the vorticity tensor, respectively. If $a=0$, we call the system (1.1) the Oldroyd-B model without damping mechanism which we investigate in this paper. The Oldroyd-B model is a typical prototypical model for viscoelastic fluids, which describes the motion of some viscoelastic flows. For more detailed physical background and derivations about this model, one refers to [2, 7, 23].

The well-posedness of the system (1.1) had been studied extensively. In the the case of $a>0$, Guillopé and Saut [19] proved that the strong solutions are local well-posed in the Sobolev space $H^{s}$. They [20] also showed that these solutions are global if the coupling parameter and the initial data are small enough. Their results were extended to the

Date: October 16, 2018.

2000 Mathematics Subject Classification. 35Q30, 76D05; 35B40.

Key words and phrases. incompressible Oldroyd-B model, critical spaces, global solution. 
$L^{p}$ framework by Fernandez-Cara, Guillén and Ortega [15]. Chemin and Masmoudi [7] initiated the study of the global existence and uniqueness in the critical Besov spaces, and their results were improved later by Zi, Fang and Zhang [16] to the case of the non-small coupling parameter. For more results on the well-posedness and the blow-up criterion, one refers to [7, 8, 16, 17, 22] and references therein.

Now let us say a few words about the so-called critical spaces, for the incompressible Navier-Stokes equations

$$
(\mathrm{INS}) \quad\left\{\begin{array}{l}
u_{t}+u \cdot \nabla u-\nu \Delta u+\nabla p=0, \\
\nabla \cdot u=0 \\
u(0, x)=u_{0}(x)
\end{array}\right.
$$

if $(u(t, x), p(t, x))$ is a solution of (INS), then for $\lambda>0$

$$
\left(u_{\lambda}(t, x), p_{\lambda}(t, x)\right) \triangleq\left(\lambda u\left(\lambda^{2} t, \lambda x\right), \lambda^{2} p\left(\lambda^{2} t, \lambda x\right)\right),
$$

is also a solution of (INS). Moreover, the functional space $X$ is called critical to the system (INS) if the corresponding norm is invariant under the scaling (1.2). It is Obvious that $\dot{H}^{\frac{d}{2}-1}$ is a critical space. Fujita and Kato [18] proved the wellposedness of (INS) in $\dot{H}^{\frac{d}{2}-1}$, see also [3, 4, 5, 6] and references therein for the other critical spaces. Although the system (1.1) does not have any scaling invariance, one may find that if the coupling term $\nabla \cdot \tau$ as well as the damping term $\tau$ is neglected, and $(u, \tau)$ is a solution of (1.1), then for $\lambda>0$

$$
\left(u_{\lambda}(t, x), \tau_{\lambda}(t, x), p_{\lambda}(t, x)\right) \triangleq\left(\lambda u\left(\lambda^{2} t, \lambda x\right), \tau\left(\lambda^{2} t, \lambda x\right), \lambda^{2} p\left(\lambda^{2} t, \lambda x\right)\right)
$$

is also a solution of (1.1). This leads us to define the following critical spaces of the system (1.1) as in [7, 16],

Definition 1.1. A functional space is called critical to (1.1) if the associated norm is invariant under the transformation $(u, \tau) \rightarrow\left(u_{\lambda}, \tau_{\lambda}\right)$ (up to a constant independent of $\lambda$ ).

It is obvious that $\dot{H}^{\frac{d}{2}} \times \dot{H}^{\frac{d}{2}-1}$ and $\dot{B}_{p, q}^{\frac{d}{p}} \times \dot{B}_{p, q}^{\frac{d}{p}-1}$ are critical spaces. And the reason why one has to consider the well-posedness in such spaces has been fully explained in [7].

As for the researches of other special cases of the system (1.1), we sketch some results here. If $b=0$, the existence of the global weak solution had been proved by Lions and Masmoudi [21]. If $\mu=0$ and the equation of $\tau$ contains viscous term $-\Delta \tau$, the global existence of small smooth solutions had been proved by Elgindi, Rousset and Liu [13, 14, and the similar result with general data had also been showed for more special models in [13]. When $a=0$, Zhu Yi [24] constructed a global smooth solution in $3 D$ very recently. More precisely,

Theorem 1.1. 24] Let $\nu, \mu_{1}, \mu_{2}>0$ and $a=0$. Suppose that $\nabla \cdot u=0,\left(\tau_{0}\right)^{i, j}=\left(\tau_{0}\right)^{j, i}$ and initial data $\Lambda^{-1} u_{0}, \Lambda^{-1} \tau_{0} \in H^{3}\left(\mathbb{R}^{3}\right)$. Then there exists a small constant $\epsilon$ such that system (1.1) admits a unique global classical solution provided that

$$
\left\|\Lambda^{-1} u_{0}\right\|_{H^{3}}+\left\|\Lambda^{-1} \tau_{0}\right\|_{H^{3}} \leq \epsilon
$$

where $\Lambda^{-1}=(-\Delta)^{\frac{1}{2}}$. 
It is noted that the required regularity of Theorem 1.1 is far from the regularity prescribed by the scaling (1.3), which inspires us to consider the well-posedness of (1.1) $(a=0)$ in critical Besov spaces just like Chemin and Masmoudi had done in [7, 16] for $a>0$.

Now we state the main result.

Theorem 1.2. Let $\nu, \mu_{1}, \mu_{2}>0$ and $a=0$. There exists a small constant $\varepsilon$ such that if $\tau_{0} \in \dot{B}_{2,1}^{\frac{d}{2}-1} \cap \dot{B}_{2,1}^{\frac{d}{2}}, u_{0} \in \dot{B}_{2,1}^{\frac{d}{2}-1}$ with

$$
\left\|u_{0}\right\|_{\dot{B}_{2,1}^{\frac{d}{2}-1}}+\left\|\tau_{0}\right\|_{\dot{B}_{2,1}^{\frac{d}{2}-1} \cap \dot{B}_{2,1}^{\frac{d}{2}}} \leq \varepsilon
$$

then the system (1.1) has a unique global solution $(u, \tau)$ such that

$$
\begin{aligned}
& u \in C\left(\mathbb{R}^{+} ; \dot{B}_{2,1}^{\frac{d}{2}-1}\right) \cap L^{1}\left(\mathbb{R}^{+} ; \dot{B}_{2,1}^{\frac{d}{2}+1}\right) ; \\
& \tau \in C\left(\mathbb{R}^{+} ; \dot{B}_{2,1}^{\frac{d}{2}-1} \cap \dot{B}_{2,1}^{\frac{d}{2}}\right), \quad \mathbb{P} \nabla \cdot \tau \in L^{1}\left(\mathbb{R}^{+} ; \dot{B}_{2,1}^{\frac{d}{2}-1}+\dot{B}_{2,1}^{\frac{d}{2}}\right) .
\end{aligned}
$$

Here $\mathbb{P}$ is the projection operator, and $\dot{B}_{2,1}^{s}$ is Besov space. One refers to Section 0 for its definition.

Remark 1.3. When $d=3$, noting that $H^{2} \hookrightarrow \dot{B}_{2,1}^{\frac{1}{2}}$ (see Proposition 2.4), Theorem 1.2 allows us to involve a class of functions of the initial data that Theorem 1.1 does not contain. For example,

$$
\chi_{p}(D)|x|^{-\sigma}, \quad 1<\sigma \leq 3 / 2,
$$

where $\chi_{p}(D) f \triangleq \mathcal{F}^{-1}\left(\chi_{\mathcal{B}(0, p)} \hat{f}\right)$ with the radial function $\chi_{\mathcal{B}(0, p)} \in \mathcal{S}\left(\mathbb{R}^{3}\right)$ supported in the ball $\mathcal{B}=\left\{\xi \in \mathbb{R}^{d},|\xi| \leq p\right\}$. It is not difficult to check that

$$
\chi_{p}(D)|x|^{-\sigma} \in \dot{B}_{2,1}^{\frac{1}{2}}\left(\mathbb{R}^{3}\right) ; \quad \chi_{p}(D)|x|^{-\sigma} \notin H^{2}\left(\mathbb{R}^{3}\right) .
$$

For the detailed proof, one please refers to Proposition 2.6.

In [24], the author observed that $(u, \mathbb{P} \nabla \cdot \tau)$ satisfies some kind of the damped wave equations and has enough decay regardless of $a=0$, then he proved Theorem 1.1 by constructing two special time-weighted energies. However, the approach used in [24] seems not to work for the critical spaces. On the other hand, the method used in [7] for the critical Besov spaces relies heavily on the damping effect $(a>0)$. This makes us to dig out more informations about the system (1.1) when $a=0$. We find out that one part of the stress tensor $\tau$ has damping effect while the stress tensor itself does not have. More precisely, motivated by the work of Danchin [10] on the compressible Navier-Stokes equations, we study the following mixed linear system

$$
\left\{\begin{array}{l}
u_{t}-\nu \Delta u-\mu_{1} \Lambda\left(\Lambda^{-1} \mathbb{P} \nabla \cdot \tau\right)=\mathbb{P} E, \\
\left(\Lambda^{-1} \mathbb{P} \nabla \cdot \tau\right)_{t}+\frac{\mu_{2}}{2} \Lambda u=\Lambda^{-1} \mathbb{P} \nabla \cdot F,
\end{array}\right.
$$

where $\mathbb{P}$ is the projection operator,

$$
\Lambda=(-\Delta)^{\frac{1}{2}} \text { and } \Lambda^{-1}=(-\Delta)^{-\frac{1}{2}} .
$$


Let $\mathcal{G}(x, t)$ be the Green matrix of system (1.4), we derive

$$
\hat{\mathcal{G}}(\xi, t)=\left(\begin{array}{cc}
\frac{\lambda_{+} e^{\lambda_{+}{ }^{t}-\lambda_{-} e^{\lambda_{-} t}}}{\lambda_{+}-\lambda_{-}} & \mu_{1}|\xi| \frac{e^{\lambda_{+}{ }^{t}-e^{\lambda_{-} t}}}{\lambda_{+}-\lambda_{-}} \\
-\frac{\mu_{2}}{2}|\xi| \frac{e^{\lambda^{t}}-e^{\lambda_{-}} t}{\lambda_{+}-\lambda_{-}} & \frac{\lambda_{+} e^{\lambda_{-}}-\lambda_{-} e^{\lambda_{+} t}}{\lambda_{+}-\lambda_{-}}
\end{array}\right),
$$

where

$$
\begin{aligned}
& \lambda_{+}=\frac{-\nu|\xi|^{2}+\sqrt{\nu^{2}|\xi|^{4}-2 \mu_{1} \mu_{2}|\xi|^{2}}}{2}, \\
& \lambda_{-}=\frac{-\nu|\xi|^{2}-\sqrt{\nu^{2}|\xi|^{4}-2 \mu_{1} \mu_{2}|\xi|^{2}}}{2} .
\end{aligned}
$$

By analyzing the behaviors of $\widehat{\mathcal{G}}(x, t)$ in different frequencies, we discover that $u$ as well as the low frequencies of $\Lambda^{-1} \mathbb{P} \nabla \cdot \tau$ has a parabolic smoothing effect, and the high frequencies of $\Lambda^{-1} \mathbb{P} \nabla \cdot \tau$ have a damping effect. Specifically, we set the energy in the low frequencies

$$
\mathcal{E}_{r}^{l}(t) \triangleq \sup _{t}\|u\|_{\dot{B}_{2,1}^{\frac{d}{2}-1}}^{l}+\sup _{t}\left\|\Lambda^{-1} \mathbb{P} \nabla \cdot \tau\right\|_{\dot{B}_{2,1}^{\frac{d}{2}-1}}^{l}+\int_{0}^{t}\|u\|_{\dot{B}_{2,1}^{\frac{d}{2}+1}}^{l} \mathrm{~d} t^{\prime}+\int_{0}^{t}\left\|\Lambda^{-1} \mathbb{P} \nabla \cdot \tau\right\|_{\dot{B}_{2,1}^{\frac{d}{2}+1}}^{l} \mathrm{~d} t^{\prime}
$$

and the energy in the high frequencies

$$
\mathcal{E}_{r}^{h}(t) \triangleq \sup _{t}\|u\|_{\dot{B}_{2,1}^{\frac{d}{2}-1}}^{h}+\sup _{t}\|\mathbb{P} \nabla \cdot \tau\|_{\dot{B}_{2,1}^{\frac{d}{2}-1}}^{h}+\int_{0}^{t}\|u\|_{\dot{B}_{2,1}^{\frac{d}{2}+1}}^{h} \mathrm{~d} t^{\prime}+\int_{0}^{t}\|\mathbb{P} \nabla \cdot \tau\|_{\dot{B}_{2,1}^{\frac{d}{2}-1}}^{h} \mathrm{~d} t^{\prime}
$$

where $\|\cdot\|^{l}$ and $\|\cdot\|^{h}$ denotes the low and high part of corresponding norm (see Subsection 2.3). Unfortunately, there seems no damping effect on another part of the tensor $\tau$, and the following estimates do not hold

$$
\|\tau\|_{\dot{B}_{2,1}^{\frac{d}{2}-1}}^{l} \leq C\left\|\Lambda^{-1} \mathbb{P} \nabla \cdot \tau\right\|_{\dot{B}_{2,1}^{\frac{d}{2}-1}}^{l}, \quad\|\tau\|_{\dot{B}_{2,1}^{\frac{d}{2}-1}}^{l} \leq C\|\mathbb{P} \nabla \cdot \tau\|_{\dot{B}_{2,1}^{\frac{d}{2}-1}}^{h} \cdot
$$

Thus it is difficult to deal with some parts of the nonlinear terms (e.g. $Q(\tau, \nabla u))$ by the original energies $\mathcal{E}_{r}^{l}(t), \mathcal{E}_{r}^{h}(t)$. Naturally, we want to get more estimates containing term $\tau$ instead of $\mathbb{P} \nabla \cdot \tau$, and this motivates us to construct the energies $\mathcal{E}^{l}(t), \mathcal{E}^{h}(t)$ as the following,

$$
\mathcal{E}^{l}(t) \triangleq \sup _{t}\|u\|_{\dot{B}_{2,1}^{\frac{d}{2}-1}}^{l}+\sup _{t}\|\tau\|_{\dot{B}_{2,1}^{\frac{d}{2}-1}}^{l}+\int_{0}^{t}\|u\|_{\dot{B}_{2,1}^{\frac{d}{2}+1}}^{l} \mathrm{~d} t^{\prime}+\int_{0}^{t}\left\|\Lambda^{-1} \mathbb{P} \nabla \cdot \tau\right\|_{\dot{B}_{2,1}^{\frac{d}{2}+1}}^{l} \mathrm{~d} t^{\prime}
$$

and

$$
\mathcal{E}^{h}(t) \triangleq \sup _{t}\|u\|_{\dot{B}_{2,1}^{\frac{d}{2}-1}}^{h}+\sup _{t}\|\tau\|_{\dot{B}_{2,1}^{\frac{d}{2}}}^{h}+\int_{0}^{t}\|u\|_{\dot{B}_{2,1}^{\frac{d}{2}+1}}^{h} \mathrm{~d} t^{\prime}+\int_{0}^{t}\|\mathbb{P} \nabla \cdot \tau\|_{\dot{B}_{2,1}^{\frac{d}{2}-1}}^{h} \mathrm{~d} t^{\prime} .
$$

Then the above-mentioned energies supply $L_{T}^{\infty}$ estimate of $\|\tau\|_{\dot{B}_{2,1}^{\frac{d}{2}-1}}\left(\|\tau\|_{\dot{B}_{2,1}^{\frac{d}{2}}}\right)$ in the low (high) frequencies. For more details, please see Section 3. Let us emphasize that if we handle the nonlinear terms of $\tau$ involved, we shall appeal to the way used in [24], i.e.,

$$
\mathbb{P} \nabla \cdot \tau=\mathbb{P}(u \cdot \nabla \mathbb{P} \nabla \cdot \tau)+\text { some terms containing } \nabla u .
$$

Notations. Throughout this paper, $C$ stands for the constant and changes from line to line. We use $\widehat{u}$ and $\mathcal{F}(u)$ to denote the Fourier transform of $u$. 


\section{Littlewood-Paley theory and Besov spaces}

2.1. Littlewood-Paley decomposition. Now we introduce the Littlewood-Paley decomposition, which relies on the dyadic partition of unity, and we can refer to [1] for more details. Let us choose two radial functions $\varphi, \chi \in \mathcal{S}\left(\mathbb{R}^{d}\right)$ supported in $\mathcal{C}=\left\{\xi \in \mathbb{R}^{d}, \frac{3}{4} \leq\right.$ $\left.|\xi| \leq \frac{8}{3}\right\}$ and $\mathcal{B}=\left\{\xi \in \mathbb{R}^{d},|\xi| \leq \frac{4}{3}\right\}$ respectively such that

$$
\sum_{j \in \mathbb{Z}} \varphi\left(2^{-j} \xi\right)=1 \text { if } \xi \neq 0
$$

Denote $h(x)=\mathcal{F}^{-1}(\varphi(\xi))$, we define the dyadic blocks as follows

$$
\begin{aligned}
& \dot{\Delta}_{j} u=\varphi\left(2^{-j} D\right) u=2^{j d} \int_{\mathbb{R}^{d}} h\left(2^{j} y\right) u(x-y) \mathrm{d} y, \\
& \dot{S}_{j} u=\chi\left(2^{-j} D\right) u .
\end{aligned}
$$

Definition 2.1. We denote by $\mathcal{S}_{h}^{\prime}$ the space of temperate distributions u such that

$$
\lim _{j \rightarrow-\infty} \dot{S}_{j} u=0 \text { in } \mathcal{S}^{\prime}
$$

Remark 2.1. If a temperate distribution $u$ is such that its Fourier transform $\mathcal{F} u$ is locally integrable near 0 , then $u$ belongs to $\mathcal{S}_{h}^{\prime}$.

Then the homogeneous Littlewood-paley decomposition is defined as

$$
u=\sum_{j \in \mathbb{Z}} \dot{\Delta}_{j} u, \quad \text { for } \quad u \in \mathcal{S}_{h}^{\prime} .
$$

With our choice of $\varphi$ and $\chi$, it is easy to verify that

$$
\dot{\Delta}_{j} \dot{\Delta}_{k} u=0 \text { if }|j-k| \geq 2 \text {, and } \quad \dot{\Delta}_{j}\left(\dot{S}_{k-1} u \dot{\Delta}_{k} u\right)=0 \text { if }|j-k| \geq 5 .
$$

Next, let us introduce a useful lemma which will be repeatedly used throughout this paper.

Lemma 2.2. [1] Let $1 \leq p \leq q \leq+\infty$. Then for any $\gamma \in(\mathbb{N} \cup\{0\})^{d}$, there exists a constant $C$ independent of $f, j$ such that

$$
\begin{aligned}
& \operatorname{supp} \hat{f} \subseteq\left\{|\xi| \leq A_{0} 2^{j}\right\} \Rightarrow\left\|\partial^{\gamma} f\right\|_{L^{q}} \leq C 2^{j|\gamma|+j d\left(\frac{1}{p}-\frac{1}{q}\right)}\|f\|_{L^{p}}, \\
& \operatorname{supp} \hat{f} \subseteq\left\{A_{1} 2^{j} \leq|\xi| \leq A_{2} 2^{j}\right\} \Rightarrow\|f\|_{L^{p}} \leq C 2^{-j|\gamma|} \sup _{|\beta|=|\gamma|}\left\|\partial^{\beta} f\right\|_{L^{p}} .
\end{aligned}
$$

\subsection{Homogeneous Besov space.}

Definition 2.2. Let $u \in \mathcal{S}^{\prime}\left(\mathbb{R}^{d}\right), s \in \mathbb{R}$, and $1 \leq p, r \leq \infty$, we set

$$
\|u\|_{\dot{B}_{p, r}^{s}} \triangleq\left\|\left\{2^{j s}\left\|\dot{\Delta}_{j} u\right\|_{L^{p}}\right\}_{j}\right\|_{l^{r}}
$$

We then define the space $\dot{B}_{p, r}^{s} \triangleq\left\{u \in \mathcal{S}_{h}^{\prime},\|u\|_{\dot{B}_{p, r}^{s}}<\infty\right\}$.

Remark 2.3. The definition of the space $\dot{B}_{p, r}^{s}$ does not depend on the choice of the couple $(\varphi, \chi)$ defining the Littlewood-Paley decomposition.

Let us now state some classical properties of the homogeneous Besov spaces. 
Proposition 2.4. For all $s, s_{1}, s_{2} \in \mathbb{R}, 1 \leq p, p_{1}, p_{2}, r, r_{1}, r_{2} \leq+\infty$, we have the following properties:

(i) If $p_{1} \leq p_{2}, r_{1} \leq r_{2}$, then $\dot{B}_{p_{1}, r_{1}}^{s} \hookrightarrow \dot{B}_{p_{2}, r_{2}}^{s-\frac{d}{p_{1}}+\frac{d}{p_{2}}}$.

(ii) If $s_{1} \neq s_{2}$ and $\theta \in(0,1)$, then

$$
\|u\|_{\dot{B}_{p, r}^{\theta_{1}+(1-\theta) s_{2}}} \leq\|u\|_{\dot{B}_{p, r}^{s_{1}}}^{\theta}\|u\|_{\dot{B}_{p, r}^{s_{2}}}^{1-\theta} .
$$

(iii) $\dot{H}^{s} \approx \dot{B}_{2,2}^{s}$ and

$$
\frac{1}{C^{|s|+1}}\|u\|_{\dot{B}_{2,2}^{s}} \leq\|u\|_{\dot{H}^{s}} \leq C^{|s|+1}\|u\|_{\dot{B}_{2,2}^{s}} .
$$

(iv) If $s>0$, then $\dot{B}_{2,1}^{s} \cap L^{\infty}$ (especially $\dot{B}_{2,1}^{\frac{d}{2}}$ ) is an algebra.

Proposition 2.5. Let $s>0, u \in L^{\infty} \cap \dot{B}_{2,1}^{s}$ and $v \in L^{\infty} \cap \dot{B}_{2,1}^{s}$. Then $u v \in L^{\infty} \cap \dot{B}_{2,1}^{s}$ and

$$
\|u v\|_{\dot{B}_{2,1}^{s}} \lesssim\|u\|_{L^{\infty}}\|v\|_{\dot{B}_{2,1}^{s}}+\|v\|_{L^{\infty}}\|u\|_{\dot{B}_{2,1}^{s}} .
$$

Let $s_{1}, s_{2} \leq \frac{d}{2}$ such that $s_{1}+s_{2}>0, u \in \dot{B}_{2,1}^{s_{1}}$ and $v \in \dot{B}_{2,1}^{s_{2}}$. Then $u v \in \dot{B}_{2,1}^{s_{1}+s_{2}-\frac{d}{2}}$ and

$$
\|u v\|_{\dot{B}_{2,1}^{s_{1}+s_{2}-\frac{d}{2}}} \lesssim\|u\|_{\dot{B}_{2,1}^{s_{1}}}\|v\|_{\dot{B}_{2,1}^{s_{2}}} \text {. }
$$

Let $u \in \dot{B}_{d, \infty}^{0}$ and $v \in \dot{B}_{d, 1}^{1}$. Then $u v \in \dot{B}_{d, \infty}^{0}$

$$
\|u v\|_{\dot{B}_{d, \infty}^{0}} \lesssim\|u\|_{\dot{B}_{d, \infty}^{0}}\|v\|_{\dot{B}_{d, 1}^{1}} .
$$

We can refer to [1] for the proof of these propositions.

Proposition 2.6. Let $\sigma \in\left(1, \frac{3}{2}\right]$, then we get

$$
\chi_{p}(D)|x|^{-\sigma} \in \dot{B}_{2,1}^{\frac{1}{2}}\left(\mathbb{R}^{3}\right) ; \quad \chi_{p}(D)|x|^{-\sigma} \notin H^{2}\left(\mathbb{R}^{3}\right) .
$$

Here $\chi_{p}(D) f=\mathcal{F}^{-1}\left(\chi_{B(0, p)} \hat{f}\right)$.

Proof. Noticing that

$$
\mathcal{F}\left(|x|^{-\sigma}\right)=C|\xi|^{\sigma-3} \in L_{l o c}^{1}\left(\mathbb{R}^{3}\right)
$$

and Remark 2.1, we get $|x|^{-\sigma} \in \mathcal{S}_{h}^{\prime}$. By direct computations we have

$$
\begin{aligned}
\dot{\Delta}_{j}|x|^{-\sigma} & =2^{3 j} \int_{\mathbb{R}^{3}} h\left(2^{j}(x-y)\right)|y|^{-\sigma} \mathrm{d} y \\
& =2^{j \sigma} \int_{\mathbb{R}^{3}} h\left(2^{j} x-2^{j} y\right)\left|2^{j} y\right|^{-\sigma} \mathrm{d} 2^{j} y \\
& =2^{j \sigma} \widetilde{h}\left(2^{j} x\right),
\end{aligned}
$$

where $\widetilde{h}(x)=\int_{\mathbb{R}^{3}} h(x-y)|y|^{-\sigma} \mathrm{d} y$. Since $(\mathcal{F} h)(\xi)=\varphi(\xi) \in \mathcal{D}\left(\mathbb{R}^{3} /\{0\}\right)$, we obtain $\mathcal{F} \tilde{h} \in$ $\mathcal{D}\left(\mathbb{R}^{3}\right)$. This implies $\|\widetilde{h}\|_{L^{2}}<+\infty$. From above analysis, we easily get

$$
\left\|\dot{\Delta}_{j}|x|^{-\sigma}\right\|_{L^{2}}=2^{j\left(\sigma-\frac{3}{2}\right)}\|\widetilde{h}\|_{L^{2}}
$$

Combining with the definition of the Besov space and $\operatorname{supp} \chi_{p} \subset B(0, p)$, we derive that

$$
\left\|\chi_{p}(D)|x|^{-\sigma}\right\|_{\dot{B}_{2,1}^{\frac{1}{2}}} \lesssim \sum_{j \leq N_{p}} 2^{j(\sigma-1)}\|\widetilde{h}\|_{L^{2}}<\infty .
$$


However, it is obvious that

$$
\left\|\chi_{p}(D)|x|^{-\sigma}\right\|_{L^{2}}=\left\|\chi_{p}(\xi)|\xi|^{\sigma-3}\right\|_{L^{2}}=\infty .
$$

2.3. Hybrid Besov spaces. Let us now introduce the hybrid Besov spaces we will work in this paper.

Definition 2.3. [11] Let $s, t \in \mathbb{R}$. We set

$$
\|u\|_{\dot{B}^{s, t}}=\sum_{j \leq 0} 2^{j s}\left\|\dot{\Delta}_{j} u\right\|_{L^{2}}+\sum_{j>0} 2^{j t}\left\|\dot{\Delta}_{j} u\right\|_{L^{2}} .
$$

We then define the space $\dot{B}^{s, t} \triangleq\left\{u \in \mathcal{S}_{h}^{\prime},\|u\|_{\dot{B}^{s, t}}<\infty\right\}$.

We will often use the definition:

$$
\|u\|_{\dot{B}_{p, 1}^{s}}^{l} \triangleq \sum_{j \leq 0} 2^{j s}\left\|\dot{\Delta}_{j} u\right\|_{p} ; \quad\|u\|_{\dot{B}_{p, 1}^{s}}^{h} \triangleq \sum_{j>0} 2^{j s}\left\|\dot{\Delta}_{j} u\right\|_{p}
$$

We also define the norm of the space $L_{T}^{r}\left(\dot{B}^{s, t}\right)$

$$
\|u\|_{L_{T}^{r}\left(\dot{B}^{s, t}\right)}=\left(\int_{0}^{T}\|u\|_{\dot{B}^{s, t}}^{r} \mathrm{~d} t\right)^{\frac{1}{r}}
$$

with the usual change if $r=\infty$. Furthermore, the norm of the time-space Besov space $\widetilde{L}_{T}^{r}\left(\dot{B}^{s, t}\right)$ is defined by

$$
\|u\|_{\widetilde{L}_{T}^{r}\left(\dot{B}^{s, t}\right)}=\sum_{j \leq 0} 2^{j s}\left\|\dot{\Delta}_{j} u\right\|_{L_{T}^{r} L^{2}}+\sum_{j>0} 2^{j s}\left\|\dot{\Delta}_{j} u\right\|_{L_{T}^{r} L^{2}}
$$

Remark 2.7. (i) By the Minkowski inequality, we easily find that $\widetilde{L}_{T}^{1}\left(\dot{B}^{s, t}\right)=L_{T}^{1}\left(\dot{B}^{s, t}\right)$ and $\widetilde{L}_{T}^{r}\left(\dot{B}^{s, t}\right) \subseteq L_{T}^{r}\left(\dot{B}^{s, t}\right)$ for $r>1$.

(ii) The properties of Proposition 2.5 for product remain true for the time-space Besov spaces.

The following Proposition is a direct consequence of the definition of Besov space.

Proposition 2.8. [1] (i) We have $\dot{B}^{s, s}=\dot{B}_{2,1}^{s}$.

(ii) If $s \leq t$ then $\dot{B}^{s, t}=\dot{B}_{2,1}^{s} \cap \dot{B}_{2,1}^{t}$. Otherwise, $\dot{B}^{s, t}=\dot{B}_{2,1}^{s}+\dot{B}_{2,1}^{t}$.

(iii) If $s_{1} \leq s_{2}$ and $t_{1} \geq t_{2}$, then $\dot{B}^{s_{1}, t_{1}} \hookrightarrow \dot{B}^{s_{2}, t_{2}}$.

For the estimates of the product of two temperate distributions $u$ and $v$ in hybrid Besov spaces, we refer to the Appendix in section 5 .

\subsection{Smoothing properties for the linear heat equation.}

Proposition 2.9. [1] Let $s \in \mathbb{R},(p, r) \in[1,+\infty]^{2}, u_{0} \in \dot{B}_{p, r}^{s-1}$ and $f \in \widetilde{L}_{T}^{1}\left(\dot{B}_{p, r}^{s-1}\right)$. Let $u$ solve

$$
\left\{\begin{array}{l}
\partial_{t} u-\mu \Delta u=f \\
u(0, x)=u_{0}
\end{array}\right.
$$


Then $u \in \widetilde{L}_{T}^{\infty}\left(\dot{B}_{p, r}^{s-1}\right) \cap \widetilde{L}_{T}^{1}\left(\dot{B}_{p, r}^{s+1}\right)$ and the following estimate holds:

$$
\|u\|_{\widetilde{L}_{T}^{\infty}\left(\dot{B}_{p, r}^{s-1}\right)}+\mu\|u\|_{\widetilde{L}_{T}^{1}\left(\dot{B}_{p, r}^{s+1}\right)} \leq\left\|u_{0}\right\|_{\dot{B}_{p, r}^{s-1}}+C\|f\|_{\widetilde{L}_{T}^{1}\left(\dot{B}_{p, r}^{s-1}\right)} .
$$

If in addition $r<+\infty$, then $u \in C_{b}\left([0, T) ; \dot{B}_{p, r}^{s-1}\right)$.

The following two Propositions are used for the proof of the uniqueness.

Proposition 2.10. [1] Let $(p, r) \in[1,+\infty]^{2}$ and $s \in\left(-\min \left(\frac{d}{p}, \frac{d}{p^{\prime}}\right) ; 1+\frac{d}{p}\right)$. Let $u$ be a vector field such that $\nabla u$ belongs to $L^{1}\left(0, T ; \dot{B}_{p, r}^{\frac{d}{p}} \cap L^{\infty}\right)$. Suppose that $f_{0} \in \dot{B}_{p, r}^{s}, F \in L^{1}\left(0, T ; \dot{B}_{p, r}^{s}\right)$ and that $f \in L^{\infty}\left(0, T ; \dot{B}_{p, r}^{s}\right) \cap C\left([0, T] ; \mathcal{S}^{\prime}\right)$ solves

$$
\left\{\begin{array}{l}
\partial_{t} f+u \cdot \nabla f=F \\
f(0, x)=f_{0}
\end{array}\right.
$$

Let $V(t) \triangleq \int_{0}^{t}\|\nabla u\|_{\dot{B}_{p, r}^{\frac{d}{p}} \cap L^{\infty}} \mathrm{d} t^{\prime}$. There exists a constant $C$ depending only on $s, p$ and $d$, and such that the following inequality holds true for $t \in[0, T]$

$$
\|f\|_{\widetilde{L}_{t}^{\infty}\left(\dot{B}_{p, r}^{s}\right)} \leq e^{C V(t)}\left(\left\|f_{0}\right\|_{\dot{B}_{p, r}^{s}}+\int_{0}^{t} e^{-C V(t)}\left\|F\left(t^{\prime}\right)\right\|_{\dot{B}_{p, r}^{s}} \mathrm{~d} t^{\prime}\right) .
$$

If $r<+\infty$ then $f$ belongs to $C\left([0, T] ; \dot{B}_{p, r}^{s}\right)$.

Proposition 2.11. 9, 12] Let $s \in \mathbb{R}$. Then for any $1 \leq p, r \leq+\infty$ and $0<\epsilon \leq 1$, we have

$$
\|f\|_{\widetilde{L}_{T}^{r}\left(\dot{B}_{p, 1}^{s}\right)} \leq C \frac{\|f\|_{\widetilde{L}_{T}^{r}\left(\dot{B}_{p, \infty}^{s}\right)}}{\epsilon} \log \left(e+\frac{\|f\|_{\widetilde{L}_{T}^{r}\left(\dot{B}_{p, \infty}^{s-\epsilon}\right)}+\|f\|_{\tilde{L}_{T}^{r}\left(\dot{B}_{p, \infty}^{s+\epsilon}\right)}}{\|f\|_{\widetilde{L}_{T}^{r}\left(\dot{B}_{p, \infty}^{s}\right)}}\right)
$$

\section{Priori estimate}

Recalling (1.8) and (1.9), we denote

$$
\mathcal{E}(t) \triangleq \mathcal{E}^{l}(t)+\mathcal{E}^{h}(t) .
$$

We have the following priori estimate.

Proposition 3.1. Let $d \geq 2$ and $a=0$. Assume that the system (1.1) has a solution $(\tau, u)$ on $[0, T)$. Then, there exist two positive constants $C_{1}, C_{2}$ independent of $T$ such that

$$
\mathcal{E}(t) \leq C_{1} \mathcal{E}_{0}+C_{2} \mathcal{E}^{2}(t)
$$

where $\mathcal{E}_{0}=\left\|u_{0}\right\|_{\dot{B}_{2,1}^{\frac{d}{2}-1}}+\left\|\tau_{0}\right\|_{\dot{B}^{\frac{d}{2}-1, \frac{d}{2}}}$.

Before the proof of the priori estimate, let us introduce two important Lemmas.

First, we show the following rough energy estimate in frequency spaces. One refers to (1.6) and (1.7) for the definitions of $\mathcal{E}_{r}^{h}(t)$ and $\mathcal{E}_{r}^{l}(t)$. 
Lemma 3.2. Let $d \geq 2, a=0$ and $(u, \tau)$ be the solution of system (1.1) on $[0, T)$. The following estimate holds

$$
\begin{aligned}
\mathcal{E}_{r}^{l}(t)+\mathcal{E}_{r}^{h}(t) \leq & C_{1} \mathcal{E}_{0}+C_{2} \int_{0}^{t} \sum_{j \leq 0} 2^{j\left(\frac{d}{2}-1\right)} \tilde{E}_{j} /\left(\left\|\dot{\Delta}_{j} u\right\|_{L^{2}}+\left\|\dot{\Delta}_{j} \Lambda^{-1} \mathbb{P} \nabla \cdot \tau\right\|_{L^{2}}\right) \mathrm{d} t^{\prime} \\
& +C_{2} \int_{0}^{t} \sum_{j>0} 2^{j\left(\frac{d}{2}-1\right)}\left(\tilde{F}_{j} /\left(\left\|\dot{\Delta}_{j} u\right\|_{L^{2}}+\left\|\dot{\Delta}_{j} \mathbb{P} \nabla \cdot \tau\right\|_{L^{2}}\right)+\tilde{R}_{j} /\left\|\dot{\Delta}_{j} u\right\|_{L^{2}}\right) \mathrm{d} t^{\prime},
\end{aligned}
$$

where $C_{1}, C_{2}$ independent of $T$.

Here we shall omit the definitions of $\tilde{E}_{j}, \tilde{F}_{j}, \tilde{R}_{j}$ for their specific forms do not affect the proof of Lemma 3.2. One can refer to the proof of Proposition 3.1 for their definitions.

Proof. Let us first fix a constant $j_{0} \in \mathbb{Z}$, which will be chosen in Step 2. Throughout this part we shall suppose that $j \leq j_{0}$ as low frequencies and $j>j_{0}$ as high frequencies. Moreover, without loss of generality, we assume that

$$
\left\|\dot{\Delta}_{j} u\right\|_{L^{2}},\left\|\dot{\Delta}_{j} \Lambda^{-1} \mathbb{P} \nabla \cdot \tau\right\|_{L^{2}},\left\|\dot{\Delta}_{j} \mathbb{P} \nabla \cdot \tau\right\|_{L^{2}} \neq 0 .
$$

Step 1: Estimate of $\mathcal{E}_{r}^{l}(t)$.

Set

$$
\widetilde{C}_{0}=2^{j_{0}} .
$$

Thanks to Lemma 2.2, for any function $f$, there exist two constants $\widetilde{C}_{1}, \widetilde{C}_{2}$ such that

$$
\widetilde{C}_{1} 2^{j}\left\|\dot{\Delta}_{j} f\right\|_{L^{p}} \leq\left\|\Lambda \dot{\Delta}_{j} f\right\|_{L^{p}} \leq \widetilde{C}_{2} 2^{j}\left\|\dot{\Delta}_{j} f\right\|_{L^{p}}
$$

Applying the operator $\dot{\Delta}_{j}$ to the system (1.4), we get

$$
\left\{\begin{array}{l}
\left(\dot{\Delta}_{j} u\right)_{t}+\nu \Lambda^{2} \dot{\Delta}_{j} u-\mu_{1} \Lambda \dot{\Delta}_{j} \Lambda^{-1} \mathbb{P} \nabla \cdot \tau=\dot{\Delta}_{j} \mathbb{P} E \\
\left(\dot{\Delta}_{j} \Lambda^{-1} \mathbb{P} \nabla \cdot \tau\right)_{t}+\frac{\mu_{2}}{2} \Lambda \dot{\Delta}_{j} u=\dot{\Delta}_{j} \Lambda^{-1} \mathbb{P} \nabla \cdot F
\end{array}\right.
$$

with

$$
E=-u \cdot \nabla u ; \quad F=-u \cdot \nabla \tau-Q(\tau, \nabla u) .
$$

For the sake of simplicity, we first suppose that $E, F=0$. Taking the $L^{2}$ scalar product of the first equation of (3.4) with $\dot{\Delta}_{j} u$, of the second with $\dot{\Delta}_{j} \Lambda^{-1} \mathbb{P} \nabla \cdot \tau$, we obtain the following two identities:

$$
\begin{aligned}
& \frac{1}{2} \frac{d}{d t}\left\|\dot{\Delta}_{j} u\right\|_{L^{2}}^{2}+\nu\left\|\Lambda \dot{\Delta}_{j} u\right\|_{L^{2}}^{2}-\mu_{1}\left(\Lambda \dot{\Delta}_{j}\left(\Lambda^{-1} \mathbb{P} \nabla \cdot \tau\right), \dot{\Delta}_{j} u\right)=0, \\
& \frac{1}{2} \frac{d}{d t}\left\|\dot{\Delta}_{j}\left(\Lambda^{-1} \mathbb{P} \nabla \cdot \tau\right)\right\|_{L^{2}}^{2}+\frac{\mu_{2}}{2}\left(\Lambda \dot{\Delta}_{j} u, \dot{\Delta}_{j} \Lambda^{-1} \mathbb{P} \nabla \cdot \tau\right)=0 .
\end{aligned}
$$

To obtain an identity involving $\left(\dot{\Delta}_{j} \mathbb{P} \nabla \cdot \tau, \dot{\Delta}_{j} u\right)$, we take the scalar product of the first equation of (3.4) with $\dot{\Delta}_{j} \mathbb{P} \nabla \cdot \tau$, apply $\Lambda$ to the second equation and take the $L^{2}$ scalar product with $\dot{\Delta}_{j} u$, then sum up both equalities, which produces

$$
\frac{d}{d t}\left(\dot{\Delta}_{j} \mathbb{P} \nabla \cdot \tau, \dot{\Delta}_{j} u\right)+\nu\left(\dot{\Delta}_{j} \Lambda^{2} u, \dot{\Delta}_{j} \mathbb{P} \nabla \cdot \tau\right)-\mu_{1}\left\|\dot{\Delta}_{j} \mathbb{P} \nabla \cdot \tau\right\|_{L^{2}}^{2}+\frac{\mu_{2}}{2}\left\|\Lambda \dot{\Delta}_{j} u\right\|_{L^{2}}^{2}=0 .
$$


Calculating $\frac{\mu_{2}}{2}(3.5)+\mu_{1}(3.6)-K_{1}(3.7)$, we obtain

$$
\begin{aligned}
& \frac{1}{2} \frac{d}{d t}\left(\frac{\mu_{2}}{2}\left\|\dot{\Delta}_{j} u\right\|_{L^{2}}^{2}+\mu_{1}\left\|\dot{\Delta}_{j} \Lambda^{-1} \mathbb{P} \nabla \cdot \tau\right\|_{L^{2}}^{2}-2 K_{1}\left(\dot{\Delta}_{j} \mathbb{P} \nabla \cdot \tau, \dot{\Delta}_{j} u\right)\right) \\
& +\left(\frac{\mu_{2} \nu}{2}-\frac{\mu_{2} K_{1}}{2}\right)\left\|\Lambda \dot{\Delta}_{j} u\right\|_{L^{2}}^{2}+\mu_{1} K_{1}\left\|\dot{\Delta}_{j} \mathbb{P} \nabla \cdot \tau\right\|_{L^{2}}^{2}-\nu K_{1}\left(\dot{\Delta}_{j} \Lambda^{2} u, \dot{\Delta}_{j} \mathbb{P} \nabla \cdot \tau\right) \\
& =0 .
\end{aligned}
$$

Using Hölder's inequality, Young's inequality and (3.3), we find that for all $\epsilon_{0}, \epsilon_{1}>0$,

$$
\begin{aligned}
& 2 K_{1}\left|\left(\dot{\Delta}_{j} \mathbb{P} \nabla \cdot \tau, \dot{\Delta}_{j} u\right)\right| \leq \frac{1}{\epsilon_{0}}\left\|\dot{\Delta}_{j} u\right\|_{2}^{2}+\epsilon_{0} \widetilde{C}_{2}^{2} \widetilde{C}_{0}^{2} K_{1}^{2}\left\|\dot{\Delta}_{j} \Lambda^{-1} \mathbb{P} \nabla \cdot \tau\right\|_{L^{2}}^{2}, \\
& \nu K_{1}\left|\left(\dot{\Delta}_{j} \Lambda^{2} u, \dot{\Delta}_{j} \mathbb{P} \nabla \cdot \tau\right)\right| \leq \frac{\nu \widetilde{C}_{0}^{2} \widetilde{C}_{2}^{2} K_{1}}{2 \epsilon_{1}}\left\|\dot{\Delta}_{j} \Lambda u\right\|_{L^{2}}^{2}+\frac{\epsilon_{1} \nu K_{1}}{2}\left\|\dot{\Delta}_{j} \mathbb{P} \nabla \cdot \tau\right\|_{L^{2}}^{2} .
\end{aligned}
$$

Inserting the above two inequalities into (3.8) with $\epsilon_{0}=\frac{4}{\mu_{2}}, \epsilon_{1}=\frac{\mu_{1}}{\nu}$, and combining (3.3), we derive

$$
\begin{aligned}
& \frac{1}{2} \frac{d}{d t}\left(\frac{\mu_{2}}{4}\left\|\dot{\Delta}_{j} u\right\|_{L^{2}}^{2}+\left(\mu_{1}-\frac{4 \widetilde{C}_{0}^{2} \widetilde{C}_{2}^{2} K_{1}^{2}}{\mu_{2}}\right)\left\|\dot{\Delta}_{j} \Lambda^{-1} \mathbb{P} \nabla \cdot \tau\right\|_{L^{2}}^{2}\right) \\
& +\left(\frac{\mu_{2} \nu}{2}-\frac{\mu_{2} K_{1}}{2}-\frac{\nu^{2} \widetilde{C}_{0}^{2} \widetilde{C}_{2}^{2} K_{1}}{2 \mu_{1}}\right) \widetilde{C}_{1}^{2} 2^{2 j}\left\|\dot{\Delta}_{j} u\right\|_{L^{2}}^{2}+\frac{\mu_{1} \widetilde{C}_{1}^{2} K_{1}}{2} 2^{2 j}\left\|\dot{\Delta}_{j} \Lambda^{-1} \mathbb{P} \nabla \cdot \tau\right\|_{L^{2}}^{2} \\
& \leq 0 .
\end{aligned}
$$

Choosing a constant $K_{1}$ such that $0<K_{1}<\min \left\{\frac{\sqrt{\mu_{1} \mu_{2}}}{2 \widetilde{C}_{0} \widetilde{C}_{2}}, \frac{\mu_{1} \mu_{2} \nu}{\mu_{1} \mu_{2}+\nu^{2} \widetilde{C}_{0}^{2} \widetilde{C}_{2}^{2}}\right\}$, we ensure that the coefficients of $\left\|\dot{\Delta}_{j} \Lambda^{-1} \mathbb{P} \nabla \cdot \tau\right\|_{L^{2}}^{2}$ and $\left\|\dot{\Delta}_{j} u\right\|_{L^{2}}^{2}$ in (3.9) are positive. Thus in the general case $(E, F \neq 0)$ that containing the nonlinear terms, we obtain

$$
\begin{aligned}
& \frac{1}{2} \frac{d}{d t}\left(\left\|\dot{\Delta}_{j} u\right\|_{L^{2}}^{2}+\left\|\dot{\Delta}_{j} \Lambda^{-1} \mathbb{P} \nabla \cdot \tau\right\|_{L^{2}}^{2}\right)+2^{2 j}\left(\left\|\dot{\Delta}_{j} u\right\|_{L^{2}}^{2}+\left\|\dot{\Delta}_{j} \Lambda^{-1} \mathbb{P} \nabla \cdot \tau\right\|_{L^{2}}^{2}\right) \\
& \leq C_{2} \tilde{E}_{j}
\end{aligned}
$$

for the constant $C_{2}>0$. Dividing by $\left\|\dot{\Delta}_{j} u\right\|_{L^{2}}+\left\|\dot{\Delta}_{j} \Lambda^{-1} \mathbb{P} \nabla \cdot \tau\right\|_{L^{2}}$, (3.10) can be written as

$$
\begin{aligned}
& \frac{d}{d t}\left(\left\|\dot{\Delta}_{j} u\right\|_{L^{2}}+\left\|\dot{\Delta}_{j} \Lambda^{-1} \mathbb{P} \nabla \cdot \tau\right\|_{L^{2}}\right)+2^{2 j}\left(\left\|\dot{\Delta}_{j} u\right\|_{L^{2}}+\left\|\dot{\Delta}_{j} \Lambda^{-1} \mathbb{P} \nabla \cdot \tau\right\|_{L^{2}}\right) \\
& \leq C_{2} \tilde{E}_{j} /\left(\left\|\dot{\Delta}_{j} u\right\|_{L^{2}}+\left\|\dot{\Delta}_{j} \Lambda^{-1} \mathbb{P} \nabla \cdot \tau\right\|_{L^{2}}\right) .
\end{aligned}
$$

Multiplying both sides of (3.11) by $2^{j\left(\frac{d}{2}-1\right)}$, summing up by $j \leq j_{0}$ (actually we can choose $j_{0}=0$, see Step 2), and then performing the time integration over $[0, t]$, we conclude that

$$
\begin{aligned}
\mathcal{E}_{r}^{l}(t) \leq & C_{1}\left(\left\|u_{0}\right\|_{\dot{B}_{2,1}^{\frac{d}{2}-1}}^{l}+\left\|\tau_{0}\right\|_{\dot{B}_{2,1}^{\frac{d}{2}-1}}^{l}\right) \\
& +C_{2} \int_{0}^{t} \sum_{j \leq j_{0}} 2^{j\left(\frac{d}{2}-1\right)} \tilde{E}_{j} /\left(\left\|\dot{\Delta}_{j} u\right\|_{L^{2}}+\left\|\dot{\Delta}_{j} \Lambda^{-1} \mathbb{P} \nabla \cdot \tau\right\|_{L^{2}}\right) \mathrm{d} t^{\prime} .
\end{aligned}
$$

\section{Step 2: Estimate of $\mathcal{E}_{r}^{h}(t)$.}


By the similar argument as the way in the low frequencies, we first consider the linear terms of (3.4) as well. From the second equation of (3.4), we can easily obtain the following inequality:

$$
\frac{1}{2} \frac{d}{d t}\left\|\dot{\Delta}_{j} \mathbb{P} \nabla \cdot \tau\right\|_{L^{2}}^{2}+\frac{\mu_{2}}{2}\left(\dot{\Delta}_{j} \Lambda^{2} u, \dot{\Delta}_{j} \mathbb{P} \nabla \cdot \tau\right)=0
$$

Calculating $\nu(3.13)-\frac{\mu_{2}}{2}(3.7)+K_{2}(3.5)$, we derive

$$
\begin{aligned}
& \frac{1}{2} \frac{d}{d t}\left(K_{2}\left\|\dot{\Delta}_{j} u\right\|_{L^{2}}^{2}+\nu\left\|\dot{\Delta}_{j} \mathbb{P} \nabla \cdot \tau\right\|_{L^{2}}^{2}-\mu_{2}\left(\dot{\Delta}_{j} \mathbb{P} \nabla \cdot \tau, \dot{\Delta}_{j} u\right)\right) \\
& +\left(\nu K_{2}-\frac{\mu_{2}^{2}}{4}\right)\left\|\Lambda \dot{\Delta}_{j} u\right\|_{L^{2}}^{2}+\frac{\mu_{1} \mu_{2}}{2}\left\|\dot{\Delta}_{j} \mathbb{P} \nabla \cdot \tau\right\|_{L^{2}}^{2}-\mu_{1} K_{2}\left(\dot{\Delta}_{j} \mathbb{P} \nabla \cdot \tau, \dot{\Delta}_{j} u\right)=0 .
\end{aligned}
$$

It is easy to check that for all $\epsilon_{0}, \epsilon_{1}>0$, we have

$$
\begin{aligned}
& \mu_{2}\left|\left(\dot{\Delta}_{j} \mathbb{P} \nabla \cdot \tau, \dot{\Delta}_{j} u\right)\right| \leq \frac{\epsilon_{0}}{2}\left\|\dot{\Delta}_{j} u\right\|_{2}^{2}+\frac{\mu_{2}^{2}}{2 \epsilon_{0}}\left\|\dot{\Delta}_{j} \mathbb{P} \nabla \cdot \tau\right\|_{L^{2}}^{2}, \\
& \mu_{1} K_{2}\left|\left(\dot{\Delta}_{j} \mathbb{P} \nabla \cdot \tau, \dot{\Delta}_{j} u\right)\right| \leq \frac{\epsilon_{1} \mu_{1} K_{2}}{2}\left\|\dot{\Delta}_{j} u\right\|_{L^{2}}^{2}+\frac{\mu_{1} K_{2}}{2 \epsilon_{1}}\left\|\dot{\Delta}_{j} \mathbb{P} \nabla \cdot \tau\right\|_{L^{2}}^{2} .
\end{aligned}
$$

Noticing that $j \geq j_{0}+1$, the following inequality shows that the smoothing effect of system (1.4) on $u$ will lose,

$$
\left\|\Lambda \dot{\Delta}_{j} u\right\|_{L^{2}}^{2} \geq \widetilde{C}_{1}^{2} 2^{2 j}\left\|\dot{\Delta}_{j} u\right\|_{L^{2}}^{2} \geq \widetilde{C}_{1}^{2} \widetilde{C}_{0}^{2}\left\|\dot{\Delta}_{j} u\right\|_{L^{2}}^{2} .
$$

Combining (3.14) (3.16), and choosing

$$
K_{2}=\epsilon_{0}=\frac{\mu_{2}^{2}}{\nu} ; \quad \epsilon_{1}=\frac{2 K_{2}}{\mu_{2}} .
$$

we finally get

$$
\begin{aligned}
& \frac{1}{2} \frac{d}{d t}\left(\frac{\mu_{2}^{2}}{2 \nu}\left\|\dot{\Delta}_{j} u\right\|_{L^{2}}^{2}+\frac{\nu}{2}\left\|\dot{\Delta}_{j} \mathbb{P} \nabla \cdot \tau\right\|_{L^{2}}^{2}\right) \\
& +\left(\frac{3}{4} \mu_{2}^{2} \widetilde{C}_{1}^{2} \widetilde{C}_{0}^{2}-\frac{\mu_{1} \mu_{2}^{3}}{\nu^{2}}\right)\left\|\dot{\Delta}_{j} u\right\|_{L^{2}}^{2}+\frac{\mu_{1} \mu_{2}}{4}\left\|\dot{\Delta}_{j} \mathbb{P} \nabla \cdot \tau\right\|_{L^{2}}^{2}=0
\end{aligned}
$$

We then define $j_{0}=\left[\log _{2} \frac{2 \sqrt{3 \mu_{1} \mu_{2}}}{3 \nu \widetilde{C}_{1}}\right]+1$ to ensure that the coefficient of $\left\|\dot{\Delta}_{j} u\right\|_{2}^{2}$ in (3.17) is positive. In light of the Remark 2.3, without loss of generality, we can define $j_{0}=0$ here and later. In the general case where the nonlinear terms $E, F \neq 0$, we get

$$
\frac{1}{2} \frac{d}{d t}\left(\left\|\dot{\Delta}_{j} u\right\|_{L^{2}}^{2}+\left\|\dot{\Delta}_{j} \mathbb{P} \nabla \cdot \tau\right\|_{L^{2}}^{2}\right)+\left(\left\|\dot{\Delta}_{j} u\right\|_{L^{2}}^{2}+\left\|\dot{\Delta}_{j} \mathbb{P} \nabla \cdot \tau\right\|_{L^{2}}^{2}\right) \leq C_{2} \tilde{F}_{j}
$$

It is easy to see that the above estimate can be rewritten as

$$
\begin{gathered}
\frac{d}{d t}\left(\left\|\dot{\Delta}_{j} u\right\|_{L^{2}}+\left\|\dot{\Delta}_{j} \mathbb{P} \nabla \cdot \tau\right\|_{L^{2}}\right)+\left(\left\|\dot{\Delta}_{j} u\right\|_{L^{2}}+\left\|\dot{\Delta}_{j} \mathbb{P} \nabla \cdot \tau\right\|_{L^{2}}\right) \\
\leq C_{2} \tilde{F}_{j} /\left(\left\|\dot{\Delta}_{j} u\right\|_{L^{2}}+\left\|\dot{\Delta}_{j} \mathbb{P} \nabla \cdot \tau\right\|_{L^{2}}\right) .
\end{gathered}
$$


Multiplying both sides of $(\underline{3.18})$ by $2^{j\left(\frac{d}{2}-1\right)}$, then summing up by $j>j_{0}$, we deduce after performing the time integration over $[0, t]$, that

$$
\begin{aligned}
& \sup _{t}\|u\|_{\dot{B}_{2,1}^{\frac{d}{2}-1}}^{h}+\int_{0}^{t}+\sup _{t}\|\mathbb{P} \nabla \cdot \tau\|_{\dot{B}_{2,1}^{\frac{d}{2}-1}}^{h}+\int_{0}^{t}\|u\|_{\dot{B}_{2,1}^{\frac{d}{2}-1}}^{h} \mathrm{~d} t^{\prime}+\|\mathbb{P} \nabla \cdot \tau\|_{\dot{B}_{2,1}^{\frac{d}{2}-1}}^{h} \mathrm{~d} t^{\prime} \\
& \lesssim\left\|u_{0}\right\|_{\dot{B}_{2,1}^{\frac{d}{2}-1}}^{h}+\left\|\tau_{0}\right\|_{\dot{B}_{2,1}^{\frac{d}{2}}}^{h}+\int_{0}^{t} \sum_{j>0} 2^{j\left(\frac{d}{2}-1\right)} \tilde{F}_{j} /\left(\left\|\dot{\Delta}_{j} u\right\|_{L^{2}}+\left\|\dot{\Delta}_{j} \mathbb{P} \nabla \cdot \tau\right\|_{L^{2}}\right) \mathrm{d} t^{\prime} .
\end{aligned}
$$

Next we show the smoothing effect on $u$. In the same way as the derivation of (3.5), we get

$$
\frac{d}{d t}\left\|\dot{\Delta}_{j} u\right\|_{L^{2}}+2^{2 j}\left\|\dot{\Delta}_{j} u\right\|_{L^{2}} \leq C\left\|\dot{\Delta}_{j} \mathbb{P} \nabla \cdot \tau\right\|_{L^{2}}+C \tilde{R}_{j} /\left\|\dot{\Delta}_{j} u\right\|_{L^{2}}
$$

Using the above inequality we deduce

$$
\begin{aligned}
\sup _{t}\|u\|_{\dot{B}_{2,1}^{\frac{d}{2}-1}}^{h}+ & \int_{0}^{t}\|u\|_{\dot{B}_{2,1}^{\frac{d}{2}+1}}^{h} \mathrm{~d} t^{\prime} \leq\left\|u_{0}\right\|_{\dot{B}_{2,1}^{\frac{d}{2}-1}}^{h}+C \int_{0}^{t}\|\mathbb{P} \nabla \cdot \tau\|_{\dot{B}_{2,1}^{\frac{d}{2}-1}}^{h} \mathrm{~d} t^{\prime} \\
& +C \int_{0}^{t} \sum_{j>0} 2^{j\left(\frac{d}{2}-1\right)} \tilde{R}_{j} /\left\|\dot{\Delta}_{j} u\right\|_{L^{2}} \mathrm{~d} t^{\prime} .
\end{aligned}
$$

Calculating (3.19) $+\eta_{1}(\underline{3.20})$, and if we choose $\eta_{1}$ small enough such that $\eta_{1} C \leq \frac{1}{2}$, then the term $\eta_{1} C \int_{0}^{t}\|\mathbb{P} \nabla \cdot \tau\|_{\dot{B}_{2,1}^{\frac{d}{2}-1}}^{h} \mathrm{~d} t^{\prime}$ can be absorbed by the left side. Therefore, we obtain

$$
\begin{aligned}
\mathcal{E}_{r}^{h}(t) \leq & C_{1}\left(\left\|u_{0}\right\|_{\dot{B}_{2,1}^{\frac{d}{2}-1}}^{h}+\left\|\tau_{0}\right\|_{\dot{B}_{2,1}^{\frac{d}{2}}}^{h}\right) \\
& +C_{2} \int_{0}^{t} \sum_{j>0} 2^{j\left(\frac{d}{2}-1\right)}\left(\tilde{F}_{j} /\left(\left\|\dot{\Delta}_{j} u\right\|_{L^{2}}+\left\|\dot{\Delta}_{j} \mathbb{P} \nabla \cdot \tau\right\|_{L^{2}}\right)+\tilde{R}_{j} /\left\|\dot{\Delta}_{j} u\right\|_{L^{2}}\right) \mathrm{d} t^{\prime} .
\end{aligned}
$$

Combining (3.12) and (3.21), we complete the proof of Lemma 3.2 .

Although we have derived the above rough energy estimate, it seems that some parts of the nonlinear terms could not be controlled by $\mathcal{E}_{r}^{h}(t)$ and $\mathcal{E}_{r}^{l}(t)$ effectively. Therefore, we have to introduce a more accurate estimate as follows.

Lemma 3.3. Let $d \geq 2, a=0$ and $(u, \tau)$ be the solution of system (1.1) on $[0, T)$. The following estimate holds

$$
\begin{aligned}
& \mathcal{E}^{l}(t)+\mathcal{E}^{h}(t) \leq C_{1} \mathcal{E}_{0} \\
& +C_{2} \int_{0}^{t} \sum_{j \leq 0} 2^{j\left(\frac{d}{2}-1\right)}\left(\tilde{E}_{j} /\left(\left\|\dot{\Delta}_{j} u\right\|_{L^{2}}+\left\|\dot{\Delta}_{j} \Lambda^{-1} \mathbb{P} \nabla \cdot \tau\right\|_{L^{2}}\right)+\tilde{Q}_{j} /\left(\left\|\dot{\Delta}_{j} u\right\|_{L^{2}}+\left\|\dot{\Delta}_{j} \tau\right\|_{L^{2}}\right)\right) \mathrm{d} t^{\prime} \\
& +C_{2} \int_{0}^{t} \sum_{j>0} 2^{j\left(\frac{d}{2}-1\right)}\left(\tilde{F}_{j} /\left(\left\|\dot{\Delta}_{j} u\right\|_{L^{2}}+\left\|\dot{\Delta}_{j} \mathbb{P} \nabla \cdot \tau\right\|_{L^{2}}\right)+\tilde{R}_{j} /\left\|\dot{\Delta}_{j} u\right\|_{L^{2}}+2^{j} \tilde{M}_{j} /\left\|\dot{\Delta}_{j} \tau\right\|_{L^{2}}\right) \mathrm{d} t^{\prime},
\end{aligned}
$$

where $C_{1}, C_{2}$ independent of $T$.

As pointed out in Lemma $\underline{3.2}$, here we also omit the definitions of $\tilde{E}_{j}, \tilde{F}_{j}, \tilde{Q}_{j}, \tilde{R}_{j}, \tilde{M}_{j}$. Please refer to the proof of Proposition 3.1 for their definitions. 
Proof. Without loss of generality, we assume

$$
\left\|\dot{\Delta}_{j} u\right\|_{L^{2}},\left\|\dot{\Delta}_{j} \Lambda^{-1} \mathbb{P} \nabla \cdot \tau\right\|_{L^{2}},\left\|\dot{\Delta}_{j} \mathbb{P} \nabla \cdot \tau\right\|_{L^{2}},\left\|\dot{\Delta}_{j} \tau\right\|_{L^{2}} \neq 0 .
$$

\section{Step 1: Estimate of $\mathcal{E}^{l}(t)$.}

In this step we will supply the estimate of $\sup _{t}\|\tau\|_{\dot{B}_{2,1}^{\frac{d}{2}-1}}^{l}$ to $\mathcal{E}_{r}^{l}(t)$.

First, let us notice some cancellations on linear terms.

$$
\begin{aligned}
& \left(\dot{\Delta}_{j} \nabla p, \dot{\Delta}_{j} u\right)=0 \\
& \left(\dot{\Delta}_{j} \nabla \cdot \tau, \dot{\Delta}_{j} u\right)+\left(\dot{\Delta}_{j} D(u), \dot{\Delta}_{j} \tau\right)=0 .
\end{aligned}
$$

A standard energy computation of system (1.1) yields

$$
\begin{aligned}
& \frac{1}{2} \frac{d}{d t}\left\|\dot{\Delta}_{j} u\right\|_{L^{2}}^{2}+\nu\left\|\Lambda \dot{\Delta}_{j} u\right\|_{L^{2}}^{2}=\mu_{1}\left(\dot{\Delta}_{j} \nabla \cdot \tau, \dot{\Delta}_{j} u\right)-\left(\dot{\Delta}_{j}(u \cdot \nabla u), \dot{\Delta}_{j} u\right) . \\
& \frac{1}{2} \frac{d}{d t}\left\|\dot{\Delta}_{j} \tau\right\|_{L^{2}}^{2}=\mu_{2}\left(\dot{\Delta}_{j} D(u), \dot{\Delta}_{j} \tau\right)-\left(\dot{\Delta}_{j}(u \cdot \nabla \tau), \dot{\Delta}_{j} \tau\right)-\left(\dot{\Delta}_{j} Q(\tau, \nabla u), \dot{\Delta}_{j} \tau\right)
\end{aligned}
$$

Calculating $\mu_{2}(3.24)+\mu_{1}(\underline{3.25})$, and thanks to (3.23) , we finally get

$$
\frac{1}{2} \frac{d}{d t}\left(\left\|\dot{\Delta}_{j} u\right\|_{L^{2}}+\left\|\dot{\Delta}_{j} \tau\right\|_{L^{2}}\right) \leq C_{2} \tilde{Q}_{j} /\left(\left\|\dot{\Delta}_{j} u\right\|_{L^{2}}+\left\|\dot{\Delta}_{j} \tau\right\|_{L^{2}}\right) .
$$

By a straightforward computation in the low frequencies, the above inequality can be estimated as

$$
\begin{aligned}
\sup _{t}\|u\|_{\dot{B}_{2,1}^{\frac{d}{2}-1}}^{l}+\sup _{t}\|\tau\|_{\dot{B}_{2,1}^{\frac{d}{2}-1}}^{l} \leq C_{1}\left(\left\|u_{0}\right\|_{\dot{B}_{2,1}^{\frac{d}{2}-1}}^{l}+\left\|\tau_{0}\right\|_{\dot{B}_{2,1}^{\frac{d}{2}-1}}^{l}\right) \\
+C_{2} \int_{0}^{t} \sum_{j \leq 0} 2^{j\left(\frac{d}{2}-1\right)} \tilde{Q}_{j} /\left(\left\|\dot{\Delta}_{j} u\right\|_{L^{2}}+\left\|\dot{\Delta}_{j} \tau\right\|_{L^{2}}\right) \mathrm{d} t^{\prime} .
\end{aligned}
$$

Combining (3.26) and (3.12), we obtain

$$
\begin{aligned}
\mathcal{E}^{l}(t) \leq & C_{1}\left(\left\|u_{0}\right\|_{\dot{B}_{2,1}^{\frac{d}{2}-1}}^{l}+\left\|\tau_{0}\right\|_{\dot{B}^{\frac{d}{2}-1, \frac{d}{2}}}^{l}\right)+C_{2} \int_{0}^{t} \sum_{j \leq 0} 2^{j\left(\frac{d}{2}-1\right)} \\
& \times\left(\tilde{E}_{j} /\left(\left\|\dot{\Delta}_{j} u\right\|_{L^{2}}+\left\|\dot{\Delta}_{j} \Lambda^{-1} \mathbb{P} \nabla \cdot \tau\right\|_{L^{2}}\right)+\tilde{Q}_{j} /\left(\left\|\dot{\Delta}_{j} u\right\|_{L^{2}}+\left\|\dot{\Delta}_{j} \tau\right\|_{L^{2}}\right)\right) \mathrm{d} t^{\prime} .
\end{aligned}
$$

Step 2: Estimate of $\mathcal{E}^{h}(t)$.

In this step we will supply the estimate of $\sup _{t}\|\tau\|_{\dot{B}_{2,1}^{\frac{d}{2}}}^{h}$ to $\mathcal{E}_{r}^{h}(t)$.

It follows from the equality (3.25) that

$$
\frac{d}{d t}\left(2^{j}\left\|\dot{\Delta}_{j} \tau\right\|_{2}\right) \leq C 2^{2 j}\left\|\dot{\Delta}_{j} u\right\|_{2}+C 2^{j} \tilde{M}_{j} /\left\|\dot{\Delta}_{j} \tau\right\|_{L^{2}}
$$

The standard computation yields

$$
\sup _{t}\|\tau\|_{\dot{B}_{2,1}^{\frac{d}{2}}}^{h} \leq\left\|\tau_{0}\right\|_{\dot{B}_{2,1}^{\frac{d}{2}}}^{h}+C \int_{0}^{t}\|u\|_{\dot{B}_{2,1}^{\frac{d}{2}+1}}^{h} \mathrm{~d} t^{\prime}+C \int_{0}^{t} \sum_{j>0} 2^{j \frac{d}{2}} \tilde{M}_{j} /\left\|\dot{\Delta}_{j} \tau\right\|_{L^{2}} \mathrm{~d} t^{\prime} .
$$


Calculating (3.21) $+\eta_{2}(3.28)$, and if we choose $\eta_{2}$ small enough such that $\eta_{2} C \leq \frac{1}{2}$, then the term $\eta_{2} C \int_{0}^{t}\|u\|_{\dot{B}_{2,1}^{2}}^{h} \frac{d}{2} \mathrm{~d} t^{\prime}$ can be absorbed by the left side, we eventually obtain

$$
\begin{aligned}
\mathcal{E}^{h}(t) \leq & C_{1}\left(\left\|u_{0}\right\|_{\dot{B}_{2,1}^{\frac{d}{2}-1}}^{h}+\left\|\tau_{0}\right\|_{\dot{B}_{2,1}^{\frac{d}{2}}}^{h}\right)+C_{2} \int_{0}^{t} \sum_{j \leq 0} 2^{j\left(\frac{d}{2}-1\right)}\left(\tilde{F}_{j} /\left(\left\|\dot{\Delta}_{j} u\right\|_{L^{2}}\right.\right. \\
& \left.\left.+\left\|\dot{\Delta}_{j} \mathbb{P} \nabla \cdot \tau\right\|_{L^{2}}\right)+\tilde{R}_{j} /\left\|\dot{\Delta}_{j} u\right\|_{L^{2}}+2^{j} \tilde{M}_{j} /\left\|\dot{\Delta}_{j} \tau\right\|_{L^{2}}\right) \mathrm{d} t^{\prime} .
\end{aligned}
$$

Combining (3.27) and (3.29), we complete the proof of Lemma 3.3.

Now let us turn to the proof of Proposition 3.1.

Proof. We will apply Lemma 3.3 to prove (3.2). Let us give the definitions of the nonlinear terms in Lemma 3.3 , the term $\tilde{E}_{j}=\tilde{E}_{j}^{1}+\tilde{E}_{j}^{2}+\tilde{E}_{j}^{3}$ with

$$
\begin{aligned}
\tilde{E}_{j}^{1}= & -\frac{\mu_{2}}{2}\left(\dot{\Delta}_{j} \mathbb{P}(u \cdot \nabla u), \dot{\Delta}_{j} u\right)-\mu_{1}\left(\dot{\Delta}_{j} \Lambda^{-1} \mathbb{P} \nabla \cdot Q(\tau, \nabla u), \dot{\Delta}_{j} \Lambda^{-1} \mathbb{P} \nabla \cdot \tau\right) \\
& +K_{1}\left(\dot{\Delta}_{j} \mathbb{P} \nabla \cdot Q(\tau, \nabla u), \dot{\Delta}_{j} u\right), \\
\tilde{E}_{j}^{2}= & -\mu_{1}\left(\dot{\Delta}_{j} \Lambda^{-1} \mathbb{P} \nabla \cdot(u \cdot \nabla \tau), \dot{\Delta}_{j} \Lambda^{-1} \mathbb{P} \nabla \cdot \tau\right), \\
\tilde{E}_{j}^{3}= & K_{1}\left(\dot{\Delta}_{j} \mathbb{P}(u \cdot \nabla u), \dot{\Delta}_{j} \mathbb{P} \nabla \cdot \tau\right)+K_{1}\left(\dot{\Delta}_{j} \mathbb{P} \nabla \cdot(u \cdot \nabla \tau), \dot{\Delta}_{j} u\right),
\end{aligned}
$$

and the term $\tilde{F}_{j}=\tilde{F}_{j}^{1}+\tilde{F}_{j}^{2}+\tilde{F}_{j}^{3}$ with

$$
\begin{aligned}
\tilde{F}_{j}^{1}= & -\nu\left(\dot{\Delta}_{j} \mathbb{P} \nabla \cdot Q(\tau, \nabla u), \dot{\Delta}_{j} \mathbb{P} \nabla \cdot \tau\right)+\frac{\mu_{2}}{2}\left(\dot{\Delta}_{j} \mathbb{P} \nabla \cdot Q(\tau, \nabla u), \dot{\Delta}_{j} u\right) \\
& -\frac{\mu_{2}^{2}}{\nu}\left(\dot{\Delta}_{j} \mathbb{P}(u \cdot \nabla u), \dot{\Delta}_{j} u\right), \\
\tilde{F}_{j}^{2}= & -\nu\left(\dot{\Delta}_{j} \mathbb{P} \nabla \cdot(u \cdot \nabla \tau), \dot{\Delta}_{j} \mathbb{P} \nabla \cdot \tau\right), \\
\tilde{F}_{j}^{3}= & \frac{\mu_{2}}{2}\left(\dot{\Delta}_{j} \mathbb{P}(u \cdot \nabla u), \dot{\Delta}_{j} \mathbb{P} \nabla \cdot \tau\right)+\frac{\mu_{2}}{2}\left(\dot{\Delta}_{j} \mathbb{P} \nabla \cdot(u \cdot \nabla \tau), \dot{\Delta}_{j} u\right),
\end{aligned}
$$

and

$$
\begin{aligned}
& \tilde{Q}_{j}=-\mu_{2}\left(\dot{\Delta}_{j}(u \cdot \nabla u), \dot{\Delta}_{j} u\right)-\mu_{1}\left(\dot{\Delta}_{j}(u \cdot \nabla \tau), \dot{\Delta}_{j} \tau\right)-\mu_{1}\left(\dot{\Delta}_{j} Q(\tau, \nabla u), \dot{\Delta}_{j} \tau\right), \\
& \tilde{R}_{j}=-\left(\dot{\Delta}_{j} \mathbb{P}(u \cdot \nabla u), \dot{\Delta}_{j} u\right), \\
& \tilde{M}_{j}=-\left(\dot{\Delta}_{j}(u \cdot \nabla \tau), \dot{\Delta}_{j} \tau\right)-\left(\dot{\Delta}_{j} Q(\tau, \nabla u), \dot{\Delta}_{j} \tau\right),
\end{aligned}
$$

for the constant $K_{1}$ is chosen in (3.9) in Lemma 3.2 .

Step 1: Estimate for $\tilde{E}_{j}^{1}, \tilde{F}_{j}^{1}, \tilde{Q}_{j}, \tilde{M}_{j}, \tilde{R}_{j}$.

First we consider the terms of $\tilde{E}_{j}^{1}, \tilde{F}_{j}^{1}, \tilde{Q}_{j}, \tilde{M}_{j}, \tilde{R}_{j}$ excluding $Q(\tau, \nabla u)$. Noting that $\nabla \cdot u=0$, we have

$$
\left(\dot{\Delta}_{j} \mathbb{P}(u \cdot \nabla u), \dot{\Delta}_{j} u\right)=\left(\dot{\Delta}_{j}(u \cdot \nabla u), \dot{\Delta}_{j} u\right) .
$$

According to Proposition 5.1 and Proposition 5.3 in Appendix, we have

$$
\begin{aligned}
\left|\left(\dot{\Delta}_{j}(u \cdot \nabla u), \dot{\Delta}_{j} u\right)\right| & \lesssim c_{j} 2^{-j\left(\frac{d}{2}-1\right)}\|u\|_{\dot{B}_{2,1}^{\frac{d}{2}+1}}\|u\|_{\dot{B}_{2,1}^{\frac{d}{2}-1}}\left\|\dot{\Delta}_{j} u\right\|_{L^{2}}, \\
\left|\left(\dot{\Delta}_{j}(u \cdot \nabla \tau), \dot{\Delta}_{j} \tau\right)\right| & \lesssim c_{j} 2^{-j \psi^{\frac{d}{2}-1, \frac{d}{2}}(j)}\|u\|_{\dot{B}_{2,1}^{\frac{d}{2}+1}}\|\tau\|_{\dot{B}^{\frac{d}{2}-1, \frac{d}{2}}}\left\|\dot{\Delta}_{j} \tau\right\|_{L^{2}},
\end{aligned}
$$


with $\sum_{j \in \mathbb{Z}} c_{j} \leq 1$. Next we consider the terms of $\tilde{E}_{j}^{1}, \tilde{F}_{j}^{1}, \tilde{Q}_{j}, \tilde{R}_{j}, \tilde{M}_{j}$ including $Q(\tau, \nabla u)$. Applying Lemma 2.2 and Hölder's inequality, for $j \leq 0$, we have

$$
\begin{aligned}
& \left|-\mu_{1}\left(\dot{\Delta}_{j} \Lambda^{-1} \mathbb{P} \nabla \cdot Q(\tau, \nabla u), \dot{\Delta}_{j} \Lambda^{-1} \mathbb{P} \nabla \cdot \tau\right)+K_{1}\left(\dot{\Delta}_{j} \mathbb{P} \nabla \cdot Q(\tau, \nabla u), \dot{\Delta}_{j} u\right)\right| \\
& \quad \lesssim\left(1+2^{j}\right)\|Q(\tau, \nabla u)\|_{L^{2}}\left(\left\|\dot{\Delta}_{j} u\right\|_{L^{2}}+\left\|\dot{\Delta}_{j} \Lambda^{-1} \mathbb{P} \nabla \cdot \tau\right\|_{L^{2}}\right) \\
& \quad \lesssim\|Q(\tau, \nabla u)\|_{L^{2}}\left(\left\|\dot{\Delta}_{j} u\right\|_{L^{2}}+\left\|\dot{\Delta}_{j} \Lambda^{-1} \mathbb{P} \nabla \cdot \tau\right\|_{L^{2}}\right) \\
& \left|-\mu_{1}\left(\dot{\Delta}_{j} Q(\tau, \nabla u), \dot{\Delta}_{j} \tau\right)\right| \lesssim\|Q(\tau, \nabla u)\|_{L^{2}}\left\|\dot{\Delta}_{j} \tau\right\|_{L^{2}}
\end{aligned}
$$

and $j \geq 0$, we have

$$
\begin{aligned}
& \left|-\nu\left(\dot{\Delta}_{j} \mathbb{P} \nabla \cdot Q(\tau, \nabla u), \dot{\Delta}_{j} \mathbb{P} \nabla \cdot \tau\right)+\frac{\mu_{2}}{2}\left(\dot{\Delta}_{j} \mathbb{P} \nabla \cdot Q(\tau, \nabla u), \dot{\Delta}_{j} u\right)\right| \\
& \lesssim 2^{j}\|Q(\tau, \nabla u)\|_{L^{2}}\left(\left\|\dot{\Delta}_{j} u\right\|_{L^{2}}+\left\|\dot{\Delta}_{j} \mathbb{P} \nabla \cdot \tau\right\|_{L^{2}}\right), \\
& \left|-\left(\dot{\Delta}_{j} Q(\tau, \nabla u), \dot{\Delta}_{j} \tau\right)\right| \lesssim\|Q(\tau, \nabla u)\|_{L^{2}}\left(\left\|\dot{\Delta}_{j} \tau\right\|_{L^{2}}+\left\|\dot{\Delta}_{j} \mathbb{P} \nabla \cdot \tau\right\|_{L^{2}}\right) .
\end{aligned}
$$

Combining (3.30) (3.33), we finally obtain

$$
\begin{aligned}
& \int_{0}^{t} \sum_{j \leq 0} 2^{j\left(\frac{d}{2}-1\right)}\left(\tilde{E}_{j}^{1} /\left(\left\|\dot{\Delta}_{j} u\right\|_{2}+\left\|\dot{\Delta}_{j} \Lambda^{-1} \mathbb{P} \nabla \cdot \tau\right\|_{L^{2}}\right)+\tilde{Q}_{j} /\left(\left\|\dot{\Delta}_{j} u\right\|_{L^{2}}+\left\|\dot{\Delta}_{j} \tau\right\|_{L^{2}}\right) \mathrm{d} t^{\prime}\right. \\
& +\int_{0}^{t} \sum_{j \leq 0} 2^{j\left(\frac{d}{2}-1\right)}\left(\tilde{F}_{j}^{1} /\left(\left\|\dot{\Delta}_{j} u\right\|_{L^{2}}+\left\|\dot{\Delta}_{j} \mathbb{P} \nabla \cdot \tau\right\|_{L^{2}}\right)+\tilde{R}_{j} /\left\|\dot{\Delta}_{j} u\right\|_{L^{2}}+2^{j} \tilde{M}_{j} /\left\|\dot{\Delta}_{j} \tau\right\|_{L^{2}}\right) \mathrm{d} t^{\prime} \\
& \lesssim\left(\sup _{t}\|\tau\|_{\dot{B}^{\frac{d}{2}-1, \frac{d}{2}}}+\sup _{t}\|u\|_{\dot{B}_{2,1}^{\frac{d}{2}-1}}\right) \int_{0}^{t}\|u\|_{\dot{B}_{2,1}^{\frac{d}{2}+1}} \mathrm{~d} t^{\prime}+\int_{0}^{t}\|Q(\tau, \nabla u)\|_{\dot{B}^{\frac{d}{2}-1, \frac{d}{2}}} \mathrm{~d} t^{\prime} \\
& \lesssim\left(\sup _{t}\|\tau\|_{\dot{B}^{\frac{d}{2}-1, \frac{d}{2}}}+\sup _{t}\|u\|_{\dot{B}_{2,1}^{\frac{d}{2}-1}}\right) \int_{0}^{t}\|u\|_{\dot{B}_{2,1}^{\frac{d}{2}+1}} \mathrm{~d} t^{\prime}
\end{aligned}
$$

where in the second inequality we use Remark 5.2 .

Step 2: Estimate for $\tilde{E}_{j}^{2}, \tilde{E}_{j}^{3}$.

Next we turn to the term $\tilde{E}_{j}^{2}$. Our strategy is to apply Proposition 5.4 and divide $\tilde{E}_{j}^{2}$ into three parts. We have $\tilde{E}_{j}^{2}=\tilde{E}_{j}^{2,1}+\tilde{E}_{j}^{2,2}+\tilde{E}_{j}^{2,3}$, where

$$
\begin{aligned}
\tilde{E}_{j}^{2,1} & =-\mu_{1}\left(\dot{\Delta}_{j} \Lambda^{-1} \mathbb{P}(u \cdot \nabla \mathbb{P} \nabla \cdot \tau), \dot{\Delta}_{j} \Lambda^{-1} \mathbb{P} \nabla \cdot \tau\right), \\
\tilde{E}_{j}^{2,2} & =-\mu_{1}\left(\dot{\Delta}_{j} \Lambda^{-1} \mathbb{P}(\nabla u \cdot \nabla \tau), \dot{\Delta}_{j} \Lambda^{-1} \mathbb{P} \nabla \cdot \tau\right), \\
\tilde{E}_{j}^{2,3} & =\mu_{1}\left(\dot{\Delta}_{j} \Lambda^{-1} \mathbb{P}\left(\nabla u \cdot \nabla \Delta^{-1} \nabla \cdot \nabla \cdot \tau\right), \dot{\Delta}_{j} \Lambda^{-1} \mathbb{P} \nabla \cdot \tau\right) .
\end{aligned}
$$

Let us consider the most difficult term $\tilde{E}_{j}^{2,1}$. We claim that

$$
\|u \otimes \mathbb{P} \nabla \cdot \tau\|_{\dot{B}_{2,1}^{\frac{d}{2}-1}}^{l} \lesssim\|u\|_{\dot{B}_{2,1}^{\frac{d}{2}-1}}\|\mathbb{P} \nabla \cdot \tau\|_{B^{\frac{d}{2}, \frac{d}{2}-1}}+\|u\|_{\dot{B}_{2,1}^{\frac{d}{2}+1}}\|\mathbb{P} \nabla \cdot \tau\|_{B^{\frac{d}{2}-2, \frac{d}{2}-1}} \cdot
$$


Then by Hölder's inequality, $\nabla \cdot u=0$ and (3.35), we obtain

$$
\begin{aligned}
& \int_{0}^{t} \sum_{j \leq 0} 2^{j\left(\frac{d}{2}-1\right)}\left|\tilde{E}_{j}^{2,1}\right| /\left(\left\|\dot{\Delta}_{j} u\right\|_{L^{2}}+\left\|\dot{\Delta}_{j} \Lambda^{-1} \mathbb{P} \nabla \cdot \tau\right\|_{L^{2}}\right) \mathrm{d} t^{\prime} \\
& \lesssim \int_{0}^{t}\|u \otimes \mathbb{P} \nabla \cdot \tau\|_{\dot{B}_{2,1}^{\frac{d}{2}-1}}^{l} \mathrm{~d} t^{\prime} \\
& \lesssim \sup _{t}\|\tau\|_{\dot{B}^{\frac{d}{2}-1, \frac{d}{2}}} \int_{0}^{t}\|u\|_{\dot{B}_{2,1}^{\frac{d}{2}+1}} \mathrm{~d} t^{\prime}+\sup _{t}\|u\|_{\dot{B}_{2,1}^{\frac{d}{2}-1}} \int_{0}^{t}\|\mathbb{P} \nabla \cdot \tau\|_{B^{\frac{d}{2}, \frac{d}{2}-1}} \mathrm{~d} t^{\prime} .
\end{aligned}
$$

The proof of (3.35) is based on the Bony's product decomposition

$$
u \otimes \mathbb{P} \nabla \cdot \tau=\dot{T}_{u} \mathbb{P} \nabla \cdot \tau+\dot{T}_{\mathbb{P} \nabla \cdot \tau} u+\dot{R}(u, \mathbb{P} \nabla \cdot \tau) .
$$

where the definitions of $\dot{T}$ and $\dot{R}$ can be referred to Appendix. Then by Proposition 5.1 , we obtain

$$
\begin{aligned}
& \left\|T_{u} \mathbb{P} \nabla \cdot \tau+T_{\mathbb{P} \nabla \cdot \tau} u\right\|_{\dot{B}_{2,1}^{\frac{d}{2}-1}}^{l} \lesssim\left\|T_{u} \mathbb{P} \nabla \cdot \tau+T_{\mathbb{P} \nabla \cdot \tau} u\right\|_{\dot{B}^{\frac{d}{2}-1, \frac{d}{2}-2}} \lesssim\|u\|_{\dot{B}_{2,1}^{\frac{d}{2}-1}}\|\mathbb{P} \nabla \cdot \tau\|_{B^{\frac{d}{2}, \frac{d}{2}-1}}, \\
& \|R(\mathbb{P} \nabla \cdot \tau, u)\|_{\dot{B}_{2,1}^{\frac{d}{2}-1}}^{l} \lesssim\|R(\mathbb{P} \nabla \cdot \tau, u)\|_{\dot{B}^{\frac{d}{2}-1, \frac{d}{2}}} \lesssim\|u\|_{\dot{B}_{2,1}^{\frac{d}{2}+1}}\|\mathbb{P} \nabla \cdot \tau\|_{B^{\frac{d}{2}-2, \frac{d}{2}-1}},
\end{aligned}
$$

which imply (3.35). Let us return to the terms $\tilde{E}_{j}^{2,2}, \tilde{E}_{j}^{2,3}$. Noticing that the incompressible condition on $u$, we have the following equalities:

$$
\begin{aligned}
& {[\nabla u \cdot \nabla \tau]^{i} \triangleq \sum_{j, k} \partial_{j} u^{k} \partial_{k} \tau^{i, j}=\sum_{j, k} \partial_{k}\left(\partial_{j} u^{k} \tau^{i, j}\right),} \\
& {\left[\nabla u \cdot \nabla \Delta^{-1} \nabla \cdot \nabla \cdot \tau\right]^{i} \triangleq \sum_{k} \partial_{i} u^{k} \partial_{k} \Delta^{-1} \nabla \cdot \nabla \cdot \tau=\sum_{k} \partial_{k}\left(\partial_{i} u^{k} \nabla \Delta^{-1} \nabla \cdot \nabla \cdot \tau\right) .}
\end{aligned}
$$

Combining (3.37) and Remark 5.2, we have

$$
\begin{aligned}
& \int_{0}^{t} \sum_{j \leq 0} 2^{j\left(\frac{d}{2}-1\right)}\left|\tilde{E}_{j}^{2,2}+\tilde{E}_{j}^{2,3}\right| /\left(\left\|\dot{\Delta}_{j} u\right\|_{L^{2}}+\left\|\dot{\Delta}_{j} \Lambda^{-1} \mathbb{P} \nabla \cdot \tau\right\|_{L^{2}}\right) \mathrm{d} t^{\prime} \\
& \lesssim \int_{0}^{t}\|\nabla u \cdot \nabla \tau\|_{\dot{B}_{2,1}^{\frac{d}{2}-2}}^{l}+\left\|\nabla u \cdot \nabla \Delta^{-1} \nabla \cdot \nabla \cdot \tau\right\|_{\dot{B}_{2,1}^{\frac{d}{2}-2}}^{l} \mathrm{~d} t^{\prime} \\
& \lesssim \int_{0}^{t}\|\nabla u \otimes \tau\|_{\dot{B}^{\frac{d}{2}-1, \frac{d}{2}}}+\left\|\nabla u \otimes \Delta^{-1} \nabla \cdot \nabla \cdot \tau\right\|_{\dot{B}^{\frac{d}{2}-1, \frac{d}{2}}} \mathrm{~d} t^{\prime} \\
& \lesssim \sup _{t}\|\tau\|_{\dot{B}^{\frac{d}{2}-1, \frac{d}{2}}} \int_{0}^{t}\|u\|_{\dot{B}_{2,1}^{\frac{d}{2}+1}} \mathrm{~d} t^{\prime}
\end{aligned}
$$

Therefore, it follows from (3.36) and (3.38) that

$$
\int_{0}^{t} \sum_{j \leq 0} 2^{j\left(\frac{d}{2}-1\right)}\left|\tilde{E}_{j}^{2}\right| /\left(\left\|\dot{\Delta}_{j} u\right\|_{L^{2}}+\left\|\dot{\Delta}_{j} \Lambda^{-1} \mathbb{P} \nabla \cdot \tau\right\|_{L^{2}}\right) \mathrm{d} t^{\prime} \lesssim \mathcal{E}^{2}(t) .
$$


Now we consider $\tilde{E}_{j}^{3}$. Applying Proposition 5.4, we have $\tilde{E}_{j}^{3}=\tilde{E}_{j}^{3,1}+\tilde{E}_{j}^{3,2}+\tilde{E}_{j}^{3,3}$, where

$$
\begin{aligned}
& \tilde{E}_{j}^{3,1}=K_{1}\left(\left(\dot{\Delta}_{j} \mathbb{P}(u \cdot \nabla u), \dot{\Delta}_{j} \mathbb{P} \nabla \cdot \tau\right)+\left(\dot{\Delta}_{j} \mathbb{P}(u \cdot \nabla \mathbb{P} \nabla \cdot \tau), \dot{\Delta}_{j} u\right)\right), \\
& \tilde{E}_{j}^{3,2}=K_{1}\left(\dot{\Delta}_{j} \mathbb{P}(\nabla u \cdot \nabla \tau), \dot{\Delta}_{j} u\right), \\
& \tilde{E}_{j}^{3,3}=-K_{1}\left(\dot{\Delta}_{j} \mathbb{P}\left(\nabla u \cdot \nabla \Delta^{-1} \nabla \cdot \nabla \cdot \tau\right), \dot{\Delta}_{j} u\right) .
\end{aligned}
$$

For the term $\tilde{E}_{j}^{3,1}$, noticing that $j \leq 0$ and Proposition 5.3, we obtain

$$
\left|\tilde{E}_{j}^{3,1}\right| \lesssim c_{j}\|u\|_{\dot{B}_{2,1}^{\frac{d}{2}+1}}\left(2^{-j\left(\frac{d}{2}-1\right)}\|u\|_{\dot{B}_{2,1}^{\frac{d}{2}-1}}\left\|\dot{\Delta}_{j} \mathbb{P} \nabla \cdot \tau\right\|_{L^{2}}+2^{-j\left(\frac{d}{2}-2\right)}\|\mathbb{P} \nabla \cdot \tau\|_{\dot{B}^{\frac{d}{2}-2, \frac{d}{2}-1}}\left\|\dot{\Delta}_{j} u\right\|_{L^{2}}\right) .
$$

Thanks to

$$
\left\|\dot{\Delta}_{j} \mathbb{P} \nabla \cdot \tau\right\|_{L^{2}} \lesssim 2^{j}\left\|\dot{\Delta}_{j} \Lambda^{-1} \mathbb{P} \nabla \cdot \tau\right\|_{L^{2}}
$$

and $j \leq 0$, we have

$$
\begin{aligned}
& \int_{0}^{t} \sum_{j \leq 0} 2^{j\left(\frac{d}{2}-1\right)}\left|\tilde{E}_{j}^{3,1}\right| /\left(\left\|\dot{\Delta}_{j} u\right\|_{L^{2}}+\left\|\dot{\Delta}_{j} \Lambda^{-1} \mathbb{P} \nabla \cdot \tau\right\|_{L^{2}}\right) \mathrm{d} t^{\prime} \\
& \lesssim\left(\sup _{t}\|u\|_{\dot{B}_{2,1}^{\frac{d}{2}-1}}+\sup _{t}\|\tau\|_{\dot{B}^{\frac{d}{2}-1, \frac{d}{2}}}\right) \int_{0}^{t}\|u\|_{\dot{B}_{2,1}^{\frac{d}{2}+1}} \mathrm{~d} t^{\prime}
\end{aligned}
$$

Dealing with the terms $\tilde{E}_{j}^{3,2}, \tilde{E}_{j}^{3,3}$ in the same way as used in the proof of $\tilde{E}_{j}^{2,2}, \tilde{E}_{j}^{2,3}$, we have

$$
\begin{aligned}
& \int_{0}^{t} \sum_{j \leq 0} 2^{j\left(\frac{d}{2}-1\right)}\left|\tilde{E}_{j}^{3,2}+\tilde{E}_{j}^{3,3}\right| /\left(\left\|\dot{\Delta}_{j} u\right\|_{L^{2}}+\left\|\dot{\Delta}_{j} \Lambda^{-1} \mathbb{P} \nabla \cdot \tau\right\|_{L^{2}}\right) \mathrm{d} t^{\prime} \\
& \lesssim \sup _{t}\|\tau\|_{\dot{B}^{\frac{d}{2}-1, \frac{d}{2}}} \int_{0}^{t}\|u\|_{\dot{B}_{2,1}^{\frac{d}{2}+1}} \mathrm{~d} t^{\prime} .
\end{aligned}
$$

Then combining (3.40) and (3.41), we deduce that

$$
\int_{0}^{t} \sum_{j \leq 0} 2^{j\left(\frac{d}{2}-1\right)}\left|\tilde{E}_{j}^{3}\right| /\left(\left\|\dot{\Delta}_{j} u\right\|_{L^{2}}+\left\|\dot{\Delta}_{j} \Lambda^{-1} \mathbb{P} \nabla \cdot \tau\right\|_{L^{2}}\right) \mathrm{d} t^{\prime} \lesssim \mathcal{E}^{2}(t) .
$$

Step 3: Estimate for $\tilde{F}_{j}^{2}, \tilde{F}_{j}^{3}$.

Using Proposition 5.4, we divide $\tilde{F}_{j}^{2}$ into three terms

$$
\begin{aligned}
& \tilde{F}_{j}^{2,1}=-\nu\left(\dot{\Delta}_{j} \mathbb{P}(u \cdot \nabla \mathbb{P} \nabla \cdot \tau), \dot{\Delta}_{j} \mathbb{P} \nabla \cdot \tau\right), \\
& \tilde{F}_{j}^{2,2}=-\nu\left(\dot{\Delta}_{j} \mathbb{P}(\nabla u \cdot \nabla \tau), \dot{\Delta}_{j} \mathbb{P} \nabla \cdot \tau\right), \\
& \tilde{F}_{j}^{2,3}=\nu\left(\dot{\Delta}_{j} \mathbb{P}\left(\nabla u \cdot \nabla \Delta^{-1} \nabla \cdot \nabla \cdot \tau\right), \dot{\Delta}_{j} \mathbb{P} \nabla \cdot \tau\right) .
\end{aligned}
$$

By Proposition 5.3, we obtain

$$
\left|\left(\dot{\Delta}_{j}(u \cdot \nabla \mathbb{P} \nabla \cdot \tau), \dot{\Delta}_{j} \mathbb{P} \nabla \cdot \tau\right)\right| \lesssim c_{j} 2^{-j\left(\frac{d}{2}-1\right)}\|u\|_{\dot{B}_{2,1}^{\frac{d}{2}+1}}\|\mathbb{P} \nabla \cdot \tau\|_{\dot{B}^{\frac{d}{2}-2, \frac{d}{2}-1}}\left\|\dot{\Delta}_{j} \mathbb{P} \nabla \cdot \tau\right\|_{L^{2}},
$$


which implies

$$
\begin{aligned}
& \int_{0}^{t} \sum_{j>0} 2^{j\left(\frac{d}{2}-1\right)}\left|\tilde{F}_{j}^{2,1}\right| /\left(\left\|\dot{\Delta}_{j} u\right\|_{L^{2}}+\left\|\dot{\Delta}_{j} \mathbb{P} \nabla \cdot \tau\right\|_{L^{2}}\right) \mathrm{d} t^{\prime} \\
& \lesssim \sup _{t}\|\tau\|_{\dot{B}^{\frac{d}{2}-1, \frac{d}{2}}} \int_{0}^{t}\|u\|_{\dot{B}_{2,1}^{\frac{d}{2}+1}} \mathrm{~d} t^{\prime} .
\end{aligned}
$$

Then we consider $\tilde{F}_{j}^{2,2}$ and $\tilde{F}_{j}^{2,3}$. Thanks to Remark 5.2 and (3.37), we obtain

$$
\begin{aligned}
& \int_{0}^{t} \sum_{j>0} 2^{j\left(\frac{d}{2}-1\right)}\left|\tilde{F}_{j}^{2,2}+\tilde{F}_{j}^{2,3}\right| /\left(\left\|\dot{\Delta}_{j} u\right\|_{L^{2}}+\left\|\dot{\Delta}_{j} \Lambda^{-1} \mathbb{P} \nabla \cdot \tau\right\|_{L^{2}}\right) \mathrm{d} t^{\prime} \\
& \lesssim \int_{0}^{t}\|\nabla u \otimes \tau\|_{\dot{B}^{\frac{d}{2}-1, \frac{d}{2}}}+\left\|\nabla u \otimes \Delta^{-1} \nabla \cdot \nabla \cdot \tau\right\|_{\dot{B}^{\frac{d}{2}-1, \frac{d}{2}}} \mathrm{~d} t^{\prime} \\
& \lesssim \sup _{t}\|\tau\|_{\dot{B}^{\frac{d}{2}-1, \frac{d}{2}}} \int_{0}^{t}\|u\|_{\dot{B}_{2,1}^{\frac{d}{2}+1}} \mathrm{~d} t^{\prime} .
\end{aligned}
$$

Combining (3.43) and (3.44), we obtain

$$
\int_{0}^{t} \sum_{j \leq 0} 2^{j\left(\frac{d}{2}-1\right)}\left|\tilde{F}_{j}^{2}\right| /\left(\left\|\dot{\Delta}_{j} u\right\|_{L^{2}}+\left\|\dot{\Delta}_{j} \Lambda^{-1} \mathbb{P} \nabla \cdot \tau\right\|_{L^{2}}\right) \mathrm{d} t^{\prime} \lesssim \mathcal{E}^{2}(t) .
$$

For the last term $\tilde{F}_{j}^{3}$, we also have $\tilde{F}_{j}^{3}=\tilde{F}_{j}^{3,1}+\tilde{F}_{j}^{3,2}+\tilde{F}_{j}^{3,3}$, where

$$
\begin{aligned}
& \tilde{F}_{j}^{3,1}=\frac{\mu_{2}}{2}\left(\left(\dot{\Delta}_{j}(u \cdot \nabla u), \dot{\Delta}_{j} \mathbb{P} \nabla \cdot \tau\right)+\left(\dot{\Delta}_{j}(u \cdot \nabla \mathbb{P} \nabla \cdot \tau), \dot{\Delta}_{j} u\right)\right), \\
& \tilde{F}_{j}^{3,2}=\frac{\mu_{2}}{2}\left(\dot{\Delta}_{j} \mathbb{P}(\nabla u \cdot \nabla \tau), \dot{\Delta}_{j} u\right), \\
& \tilde{F}_{j}^{3,3}=-\frac{\mu_{2}}{2}\left(\dot{\Delta}_{j} \mathbb{P}\left(\nabla u \cdot \nabla \Delta^{-1} \nabla \cdot \nabla \cdot \tau\right), \dot{\Delta}_{j} u\right) .
\end{aligned}
$$

Let us first consider $\tilde{F}_{j}^{3,1}$. By Proposition 5.3 , we obtain

$$
\begin{aligned}
& \left|\left(\dot{\Delta}_{j}(u \cdot \nabla u), \dot{\Delta}_{j} \mathbb{P} \nabla \cdot \tau\right)+\left(\dot{\Delta}_{j}(u \cdot \nabla \mathbb{P} \nabla \cdot \tau), \dot{\Delta}_{j} u\right)\right| \\
& \lesssim c_{j}\|u\|_{\dot{B}_{2,1}^{\frac{d}{2}+1}}\left(2^{-j\left(\frac{d}{2}-1\right)}\|u\|_{\dot{B}_{2,1}^{\frac{d}{2}-1}}\|\mathbb{P} \nabla \cdot \tau\|_{L^{2}}+2^{-j\left(\frac{d}{2}-1\right)}\|\mathbb{P} \nabla \cdot \tau\|_{\dot{B}^{\frac{d}{2}-2, \frac{d}{2}-1}}\left\|\dot{\Delta}_{j} u\right\|_{L^{2}}\right) .
\end{aligned}
$$

Then, we have

$$
\begin{aligned}
& \int_{0}^{t} \sum_{j>0} 2^{j\left(\frac{d}{2}-1\right)}\left|\tilde{F}_{j}^{3,1}\right| /\left(\left\|\dot{\Delta}_{j} u\right\|_{L^{2}}+\left\|\dot{\Delta}_{j} \Lambda^{-1} \mathbb{P} \nabla \cdot \tau\right\|_{L^{2}}\right) \mathrm{d} t^{\prime} \\
& \lesssim\left(\sup _{t}\|u\|_{\dot{B}_{2,1}^{\frac{d}{2}-1}}+\sup _{t}\|\tau\|_{\dot{B}^{\frac{d}{2}-1, \frac{d}{2}}}\right) \int_{0}^{t}\|u\|_{\dot{B}_{2,1}^{\frac{d}{2}+1}} \mathrm{~d} t^{\prime} .
\end{aligned}
$$

Dealing with the terms $\tilde{F}_{j}^{3,2}, \tilde{F}_{j}^{3,3}$ in the same way as used in the proof of $\tilde{F}_{j}^{2,2}, \tilde{F}_{j}^{2,3}$, we have

$$
\begin{aligned}
& \int_{0}^{t} \sum_{j>0} 2^{j\left(\frac{d}{2}-1\right)}\left|\tilde{F}_{j}^{2,2}+\tilde{F}_{j}^{2,3}\right| /\left(\left\|\dot{\Delta}_{j} u\right\|_{L^{2}}+\left\|\dot{\Delta}_{j} \Lambda^{-1} \mathbb{P} \nabla \cdot \tau\right\|_{L^{2}}\right) \mathrm{d} t^{\prime} \\
& \lesssim \sup _{t}\|\tau\|_{\dot{B}^{\frac{d}{2}-1, \frac{d}{2}}} \int_{0}^{t}\|u\|_{\dot{B}_{2,1}^{\frac{d}{2}+1}} \mathrm{~d} t^{\prime} .
\end{aligned}
$$


Combining (3.46), (3.47), we gather

$$
\int_{0}^{t} \sum_{j>0} 2^{j\left(\frac{d}{2}-1\right)}\left|\tilde{F}_{j}^{3}\right| /\left(\left\|\dot{\Delta}_{j} u\right\|_{L^{2}}+\left\|\dot{\Delta}_{j} \Lambda^{-1} \mathbb{P} \nabla \cdot \tau\right\|_{L^{2}}\right) \mathrm{d} t^{\prime} \lesssim \mathcal{E}^{2}(t) .
$$

According to (3.34), (3.39), (3.42), (3.45) and (3.48), we complete the proof of Proposition 3.1 .

\section{The Global existence And the UniQueness}

This section is devoted to the proof of Theorem 1.2 .

4.1. Approximate solutions and the uniform estimates. The construction of approximate solutions is based on the following local existence theorem.

Proposition 4.1. Let $\left(u_{0}, \tau_{0}\right)$ be an initial data in $H^{s}$ with strictly greater than $\frac{d}{2}$. Then a unique strictly positive time $T$ exists so that a unique solution $(u, \tau)$ exists such that

$$
u \in C\left([0, T) ; H^{s}\right) \cap L_{l o c}^{2}\left(0, T ; H^{s+1}\right) ; \tau \in C\left([0, T) ; H^{s}\right) .
$$

Moreover, the solution $(u, \tau)$ can be continued beyond $T$ if

$$
\sup _{T}\|\tau\|_{\dot{B}^{\frac{d}{2}-1, \frac{d}{2}}}+\int_{0}^{T}\|u\|_{\dot{B}_{2,1}^{\frac{d}{2}+1}} \mathrm{~d} t^{\prime}<\infty .
$$

Proof. The proof is very similar to the Theorem 1.1 in [7], here we omit it since we only have made a slight modification of its proof.

Set $\mathcal{C}_{n} \triangleq\left\{\xi \in \mathbb{R}^{d}\left|n^{-1} \leq\right| \xi \mid \leq n\right\}$. We build the approximate solution $\left(u^{n}, \tau^{n}\right)$ solving the system

$$
\left\{\begin{array}{l}
u_{t}^{n}+u^{n} \cdot \nabla u^{n}-\nu \Delta u^{n}-\nabla p=\mu_{1} \nabla \cdot \tau^{n} \\
\tau_{t}^{n}+u^{n} \cdot \nabla \tau^{n}+Q\left(\tau^{n}, \nabla u^{n}\right)=\mu_{2} D\left(u^{n}\right), \\
\nabla \cdot u^{n}=0, \\
u^{n}(0, x)=J_{n} u_{0} ; \quad \tau^{n}(0, x)=J_{n} \tau_{0},
\end{array}\right.
$$

where $\mathcal{F}\left(J_{n} U\right)(\xi)=I_{\mathcal{C}_{n}}(\xi) \mathcal{F} U(\xi)$ with $I_{\mathcal{C}_{n}}$ the smooth cut-off functions supported in $\mathcal{C}_{n}$. Using the direct computations, we gather that

$$
\lim _{n \rightarrow \infty}\left\|J_{n} u_{0}-u_{0}\right\|_{\dot{B}_{2,1}^{\frac{d}{2}-1}}=0 ; \quad \lim _{n \rightarrow \infty}\left\|J_{n} \tau_{0}-\tau_{0}\right\|_{\dot{B}^{\frac{d}{2}-1, \frac{d}{2}}}=0 .
$$

Indeed, it is easy to see that $J_{n} u_{0}, J_{n} \tau_{0} \in H^{s}$ for all $s>0$. Therefore, applying Proposition 4.1, we can obtain that there exists a maximal existence time $T_{n}>0$ such that for all $s>0$ the system (4.1) has a unique solution $\left(u^{n}, \tau^{n}\right)$,

$$
u^{n} \in C\left(\left[0, T_{n}\right) ; H^{s}\right) \cap L_{l o c}^{2}\left(0, T_{n} ; H^{s+1}\right) ; \quad \tau^{n} \in C\left(\left[0, T_{n}\right) ; H^{s}\right) .
$$

Using the definition of the Besov space, it is easy to check that

$$
\begin{aligned}
& u^{n} \in C\left(\left[0, T_{n}\right) ; \dot{B}_{2,1}^{\frac{d}{2}-1}\right) \cap L_{l o c}^{1}\left(0, T_{n} ; \dot{B}_{2,1}^{\frac{d}{2}+1}\right) ; \\
& \tau^{n} \in C\left(\left[0, T_{n}\right) ; \dot{B}^{\frac{d}{2}-1, \frac{d}{2}}\right), \quad \mathbb{P} \nabla \cdot \tau^{n} \in L_{l o c}^{1}\left(0, T_{n} ; \dot{B}^{\frac{d}{2}, \frac{d}{2}-1}\right) .
\end{aligned}
$$


Let us define

$$
T_{n}^{*}=\sup \left\{t \in\left[0, T_{n}\right) \mid \mathcal{E}^{n}(t) \leq \widetilde{C} \mathcal{E}_{0}\right\} .
$$

Firstly, we claim that

$$
T_{n}^{*}=T_{n} .
$$

Using the continuity argument, it suffices to show that for all $n \in \mathbb{N}$,

$$
\mathcal{E}^{n}(t) \leq \frac{1}{2} \widetilde{C} \mathcal{E}_{0}
$$

In fact, set $\widetilde{C}=4 C_{1}$, and choose $\mathcal{E}_{0}$ small enough such that $\mathcal{E}_{0} \leq \frac{1}{16 C_{1} C_{2}}$, then combine Proposition 3.1, we obtain

$$
\mathcal{E}^{n}(t) \leq\left(C_{1}+16 C_{1}^{2} C_{2} \mathcal{E}_{0}\right) \mathcal{E}_{0} \leq \frac{1}{2} \widetilde{C} \mathcal{E}_{0}
$$

In conclusion, we construct a sequence of approximate solution $\left(u^{n}, \tau^{n}\right)$ on $\left[0, T_{n}\right)$ satisfying

$$
\mathcal{E}^{n}\left(T_{n}\right) \leq 4 C_{1} \mathcal{E}_{0}
$$

for any $n \in \mathbb{N}$. Thanks to (4.3), we can easily obtain that $T_{n}=\infty$ by a direct application of Proposition 4.1. To sum up, we get that for all $t>0$, we have

$$
\mathcal{E}^{n}(t) \leq 4 C_{1} \mathcal{E}_{0}
$$

4.2. The existence. In this part we will use a standard compact argument to show that, up to an extraction, the sequence $\left(\left(u^{n}, \tau^{n}\right)\right)_{n \in \mathbb{N}}$ converges in $\mathcal{D}^{\prime}\left(\mathbb{R}^{+} \times \mathbb{R}^{d}\right)$ to a solution $(u, \tau)$ of (1.1) which has the desired regularity properties.

It is convenient to split $\left(u^{n}, \tau^{n}\right)$ into linear part and discrepant part. More precisely, we denote by $\left(u_{L}^{n}, \tau_{L}^{n}\right)$ the solution to

$$
\left\{\begin{array}{l}
\partial_{t} u_{L}^{n}-\nu \Delta u_{L}^{n}-\mu_{1} \nabla \cdot \tau_{L}^{n}-\nabla p=0 \\
\partial_{t} \tau_{L}^{n}-\mu_{2} D\left(u_{L}^{n}\right)=0 \\
\nabla \cdot u_{L}^{n}=0 \\
u_{L}^{n}(0, x)=J_{n} u_{0} ; \quad \tau_{L}^{n}(0, x)=J_{n} \tau_{0} .
\end{array}\right.
$$

By Proposition 3.1, we can easily get $\mathcal{E}_{L}^{n}(t) \lesssim \mathcal{E}(0)$, where $\mathcal{E}_{L}^{n}(t)$ is the energy of $\left(u_{L}^{n}, \tau_{L}^{n}\right)$ in form of (3.1). Also, we denote $\left(u_{L}, \tau_{L}\right)$ the solution to

$$
\left\{\begin{array}{l}
\partial_{t} u_{L}-\nu \Delta u_{L}-\mu_{1} \nabla \cdot \tau_{L}-\nabla p=0 \\
\partial_{t} \tau_{L}-\mu_{2} D\left(u_{L}\right)=0 \\
\nabla \cdot u_{L}=0 \\
u_{L}(0)=u_{0} ; \quad \tau_{L}(0)=\tau_{0}
\end{array}\right.
$$

It is easy to check that

$$
\begin{aligned}
& u_{L}^{n} \longrightarrow u_{L} \quad \text { in } \quad C\left(\mathbb{R}^{+} ; \dot{B}_{2,1}^{\frac{d}{2}-1}\right) \cap L^{1}\left(\mathbb{R}^{+} ; \dot{B}_{2,1}^{\frac{d}{2}+1}\right), \\
& \tau_{L}^{n} \longrightarrow \tau_{L} \quad \text { in } \quad C\left(\mathbb{R}^{+} ; \dot{B}^{\frac{d}{2}-1, \frac{d}{2}}\right) ; \quad \mathbb{P} \nabla \cdot \tau_{L}^{n} \longrightarrow \mathbb{P} \nabla \cdot \tau_{L} \quad \text { in } \quad L^{1}\left(\mathbb{R}^{+} ; \dot{B}^{\frac{d}{2}, \frac{d}{2}-1}\right) .
\end{aligned}
$$


Denote $\left(\bar{u}^{n}, \bar{\tau}^{n}\right) \triangleq\left(u^{n}-u_{L}^{n}, \tau^{n}-\tau_{L}^{n}\right)$, thanks to (4.4), we also get

$$
\begin{aligned}
& \bar{u}^{n} \in C\left(\mathbb{R}^{+} ; \dot{B}_{2,1}^{\frac{d}{2}-1}\right) \cap L^{1}\left(\mathbb{R}^{+} ; \dot{B}_{2,1}^{\frac{d}{2}+1}\right), \\
& \bar{\tau}^{n} \in C\left(\mathbb{R}^{+} ; \dot{B}^{\frac{d}{2}-1, \frac{d}{2}}\right) ; \quad \mathbb{P} \nabla \cdot \bar{\tau}^{n} \in L^{1}\left(\mathbb{R}^{+} ; \dot{B}^{\frac{d}{2}, \frac{d}{2}-1}\right) .
\end{aligned}
$$

Lemma 4.2. $\left(\left(\bar{u}^{n}, \bar{\tau}^{n}\right)\right)_{n \in \mathbb{N}}$ is uniformly bounded in $C_{\text {loc }}^{\frac{1}{2}}\left(\mathbb{R}^{+} ; \dot{B}_{2,1}^{\frac{d}{2}-2}\right) \times C_{\text {loc }}^{\frac{1}{2}}\left(\mathbb{R}^{+} ; \dot{B}_{2,1}^{\frac{d}{2}-1}\right)$.

Proof. Recall that

$$
\partial_{t} \bar{\tau}^{n}=\mu_{2} D\left(\bar{u}^{n}\right)-u^{n} \cdot \nabla \tau^{n}-Q\left(\tau^{n}, \nabla u^{n}\right),
$$

which combining with (4.4) and Proposition 5.1, we gather that

$$
\left\|\partial_{t} \bar{\tau}^{n}\right\|_{L_{T}^{2}\left(\dot{B}_{2,1}^{\frac{d}{2}-1}\right)} \lesssim\left\|\bar{u}^{n}\right\|_{L_{T}^{2}\left(\dot{B}_{2,1}^{\frac{d}{2}}\right)}+\sup _{T}\left\|\tau^{n}\right\|_{\dot{B}_{2,1}^{\frac{d}{2}}}\left\|u^{n}\right\|_{L_{T}^{2}\left(\dot{B}_{2,1}^{\frac{d}{2}}\right)}<\infty
$$

Then we get $\bar{\tau}^{n} \in C_{l o c}^{\frac{1}{2}}\left(\mathbb{R}^{+} ; \dot{B}_{2,1}^{\frac{d}{2}-1}\right)$. On the other hand,

$$
\partial_{t} \bar{u}^{n}=\mu_{1} \mathbb{P} \nabla \cdot \bar{\tau}^{n}+\nu \Delta \bar{u}^{n}+\mathbb{P} u^{n} \cdot \nabla u^{n} .
$$

Proposition 5.1 and (4.4) imply that

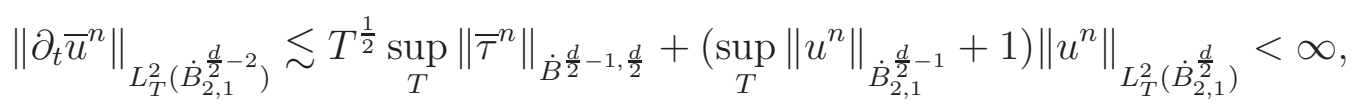

which means that $\bar{u}^{n} \in C_{l o c}^{\frac{1}{2}}\left(\mathbb{R}^{+} ; \dot{B}_{2,1}^{\frac{d}{2}-2}\right)$.

Let us choose a sequence $\left(\phi_{p}\right)_{p \in \mathbb{N}}$ of smooth cut-off functions supported in the ball $B(0, p+1)$ of $\mathbb{R}^{d}$ and equal to 1 in a neighborhood of $B(0, p)$. Lemma 4.2 ensures that

$$
\left(\phi_{p} \bar{u}^{n}, \phi_{p} \bar{\tau}^{n}\right) \text { is uniformly equicontinuous in } C\left([0, p] ; \dot{B}_{2,1}^{\frac{d}{2}-2} \times \dot{B}_{2,1}^{\frac{d}{2}-1}\right) \text {. }
$$

We know that when $s>0, \dot{B}_{2,1}^{s}(K) \cong B_{2,1}^{s}(K)$ for all compact set $K$ and $\dot{B}_{2,1}^{s} \hookrightarrow B_{2,1}^{s}$ when $s \leq 0$. Then we can get

$$
\begin{aligned}
& \left(\phi_{p} \bar{u}^{n}, \phi_{p} \bar{\tau}^{n}\right) \text { is uniformly equicontinuous in } C\left([0, p] ; B_{2,1}^{\frac{d}{2}-2} \times B_{2,1}^{\frac{d}{2}-1}\right), \\
& \left(\phi_{p} \bar{u}^{n}, \phi_{p} \bar{\tau}^{n}\right) \text { is uniformly bounded in } C\left([0, p] ; B_{2,1}^{\frac{d}{2}-1} \times B_{2,1}^{\frac{d}{2}}\right) .
\end{aligned}
$$

Moreover, we have the facts $B_{2,1}^{\frac{d}{2}-1}(K) \hookrightarrow \hookrightarrow B_{2,1}^{\frac{d}{2}-2}(K)$ and $B_{2,1}^{\frac{d}{2}}(K) \hookrightarrow \hookrightarrow B_{2,1}^{\frac{d}{2}-1}(K)$. Then, by Ascoli's Theorem and Cantor's diagonal process, we get a distribution $(\bar{u}, \bar{\tau})$ such that

$$
\left(\phi_{p} \bar{u}^{n}, \phi_{p} \bar{\tau}^{n}\right) \longrightarrow\left(\phi_{p} \bar{u}, \phi_{p} \bar{\tau}\right) \quad \text { in } \quad C\left([0, p] ; B_{2,1}^{\frac{d}{2}-2} \times B_{2,1}^{\frac{d}{2}-1}\right)
$$

Denote $u \triangleq \bar{u}+u_{L}, \tau \triangleq \bar{\tau}+\tau_{L}$, we easily have

$$
\left(u^{n}, \tau^{n}\right) \longrightarrow(u, \tau) \quad \text { in } \quad \mathcal{S}^{\prime}\left(\mathbb{R}^{d} \times \mathbb{R}^{+}\right),
$$

With the help of (4.5), (4.7) and (4.8), following the argument as in [10], it is routine to verify that $(u, \tau)$ satisfies the system (1.1) in the distribution sense. Moreover, we can infer from (4.4) that

$$
\begin{aligned}
& u \in L^{\infty}\left(\mathbb{R}^{+} ; \dot{B}_{2,1}^{\frac{d}{2}-1}\right) \cap L^{1}\left(\mathbb{R}^{+} ; \dot{B}_{2,1}^{\frac{d}{2}+1}\right) \\
& \tau \in L^{\infty}\left(\mathbb{R}^{+} ; \dot{B}^{\frac{d}{2}-1, \frac{d}{2}}\right), \mathbb{P} \nabla \cdot \tau \in L^{1}\left(\mathbb{R}^{+} ; \dot{B}^{\frac{d}{2}, \frac{d}{2}-1}\right) .
\end{aligned}
$$


At last, we have to show the properties of continuity with respect to time. The continuity of $u$ is straightforward. Indeed from the equation of $u$, we have $L_{l o c}^{1}\left(\mathbb{R}^{+} ; \dot{B}_{2,1}^{\frac{d}{2}-1}\right)$ which imply $u \in C\left(\mathbb{R}^{+} ; \dot{B}_{2,1}^{\frac{d}{2}-1}\right)$. As for $\tau$, by the same argument as in [10], we get $\tau \in C\left(\mathbb{R}^{+} ; \dot{B}^{\frac{d}{2}-1, \frac{d}{2}}\right)$.

4.3. The uniqueness. Assume $\left(u_{1}, \tau_{1}\right)$ and $\left(u_{2}, \tau_{2}\right)$ are two solutions of (1.1) with the same initial data. Denote $\delta u=u_{1}-u_{2}, \delta \tau=\tau_{1}-\tau_{2}$, then $(\delta u, \delta \tau)$ satisfies

$$
\left\{\begin{array}{l}
(\delta u)_{t}-\nu \Delta \delta u=\mu_{1} \nabla \cdot \delta \tau-u_{1} \cdot \nabla \delta u-\delta u \cdot \nabla u_{2}, \\
(\delta \tau)_{t}+u_{1} \cdot \nabla \delta \tau=\mu_{2} D(\delta u)-\delta u \cdot \nabla \tau_{2}-Q\left(\tau_{1}, \nabla \delta u\right)-Q\left(\delta \tau, \nabla u_{2}\right), \\
\nabla \cdot \delta u=0 \\
\delta u(0, x)=0 ; \delta \tau(0, x)=0 .
\end{array}\right.
$$

We shall work in the following functional spaces

$$
\mathcal{H}_{T} \triangleq\left(L^{\infty}\left(0, T ; \dot{B}_{d, \infty}^{-1}\right) \cap \widetilde{L}^{1}\left(0, T ; \dot{B}_{d, \infty}^{1}\right)\right)^{d} \times L^{\infty}\left(0, T ; \dot{B}_{d, \infty}^{0}\right) .
$$

We first state that $(\delta u, \delta \tau) \in \mathcal{H}_{T}$. From the second equation of (4.10) and Proposition 2.4, Proposition 2.5, we have

$$
\begin{aligned}
& \left\|(\delta \tau)_{t}\right\|_{L_{T}^{2}\left(\dot{B}_{d, \infty}^{0}\right)} \lesssim\left\|(\delta \tau)_{t}\right\|_{L_{T}^{2}\left(\dot{B}_{2,1}^{\frac{d}{2}-1}\right)} \\
& \lesssim\left(\left\|u_{1}\right\|_{L_{T}^{2}\left(\dot{B}_{2,1}^{\frac{d}{2}}\right)}+\left\|u_{2}\right\|_{L_{T}^{2}\left(\dot{B}_{2,1}^{\frac{d}{2}}\right)}\right)\left(1+\left\|\tau_{1}\right\|_{L_{T}^{\infty}\left(\dot{B}_{2,1}^{\frac{d}{2}}\right)}+\left\|\tau_{2}\right\|_{L_{T}^{\infty}\left(\dot{B}_{2,1}^{\frac{d}{2}}\right)}\right),
\end{aligned}
$$

which means that $\delta \tau \in C^{\frac{1}{2}}\left([0, T] ; \dot{B}_{d, \infty}^{0}\right)$. A similar discussion to $\delta \tau$ entails that $\delta u \in$ $C^{\frac{1}{2}}\left([0, T] ; \dot{B}_{d, \infty}^{-1}\right)$.

Now, let us turn to the proof of estimates for $\delta \tau$. Applying Proposition 2.10 to the second equation of (4.10), we have

$$
\begin{aligned}
\|\delta \tau\|_{\widetilde{L}_{t}^{\infty}\left(\dot{B}_{d, \infty}^{0}\right)} & \lesssim e^{C \int_{0}^{t}\left\|\nabla u_{1}\right\|_{\dot{B}_{d, 1}^{1}} \mathrm{~d} t^{\prime}} \int_{0}^{t} e^{-C \int_{0}^{t^{\prime}}\left\|\nabla u_{1}\right\|_{\dot{B}_{d, 1}^{1}}^{1} \mathrm{~d} s}\|F\|_{\dot{B}_{d, \infty}^{0}} \mathrm{~d} t^{\prime} \\
& \lesssim e^{C \int_{0}^{t}\left\|\nabla u_{1}\right\|_{\dot{B}_{d, 1}^{1}} \mathrm{~d} t^{\prime}} \int_{0}^{t}\|F\|_{\dot{B}_{d, \infty}^{0}} \mathrm{~d} t^{\prime},
\end{aligned}
$$

where $F=\mu_{2} D(\delta u)-\delta u \cdot \nabla \tau_{2}-Q(\tau, \nabla \delta u)-Q\left(\delta \tau, \nabla u_{2}\right)$. By Proposition 2.4 and Proposition 2.5, we get

$$
\begin{aligned}
\|F\|_{L_{t}^{1}\left(\dot{B}_{d, \infty}^{0}\right)} \lesssim & \left\|u_{2}\right\|_{L_{t}^{1}\left(\dot{B}_{2,1}^{\frac{d}{2}+1}\right)}\|\delta \tau\|_{L_{t}^{\infty}\left(\dot{B}_{d, \infty}^{0}\right)} \\
& +\left(1+\left\|\tau_{1}\right\|_{L_{t}^{\infty}\left(\dot{B}_{2,1}^{\frac{d}{2}}\right)}+\left\|\tau_{2}\right\|_{L_{t}^{\infty}\left(\dot{B}_{2,1}^{\frac{d}{2}}\right)}\right)\|\delta u\|_{L_{t}^{1}\left(\dot{B}_{d, 1}^{1}\right.} .
\end{aligned}
$$

We then finally obtain

$$
\begin{aligned}
\|\delta \tau\|_{L_{t}^{\infty}\left(\dot{B}_{d, \infty}^{0}\right)} \lesssim & e^{C \int_{0}^{t}\left\|\nabla u_{1}\right\|_{\dot{B}_{2,1}^{\frac{d}{2}}} \mathrm{~d} t^{\prime}}\left(\left\|u_{2}\right\|_{L_{t}^{1}\left(\dot{B}_{2,1}^{\frac{d}{2}+1}\right)}\|\delta \tau\|_{L_{t}^{\infty}\left(\dot{B}_{d, \infty}^{0}\right)}+\left(1+\left\|\tau_{1}\right\|_{L_{t}^{\infty}\left(\dot{B}_{2,1}^{\frac{d}{2}}\right)}\right.\right. \\
& \left.\left.+\left\|\tau_{2}\right\|_{L_{t}^{\infty}\left(\dot{B}_{2,1}^{\frac{d}{2}}\right)}\right)\|\delta u\|_{L_{t}^{1}\left(\dot{B}_{d, 1}^{1}\right)}\right) .
\end{aligned}
$$


From Proposition 2.11, we infer

$$
\|\delta u\|_{L_{t}^{1}\left(\dot{B}_{d, 1}^{1}\right)} \lesssim\|\delta u\|_{\widetilde{L}_{t}^{1}\left(\dot{B}_{d, \infty}^{1}\right)} \log \left(e+\frac{\|\delta u\|_{\widetilde{L}_{t}^{1}\left(\dot{B}_{d, \infty}^{0}\right)}+\|\delta u\|_{\widetilde{L}_{t}^{1}\left(\dot{B}_{d, \infty}^{2}\right)}}{\|\delta u\|_{\widetilde{L}_{t}^{1}\left(\dot{B}_{d, \infty}^{1}\right)}}\right) .
$$

Taking $T$ small enough such that $\left\|u_{2}\right\|_{L_{t}^{1}\left(\dot{B}_{2,1}^{\frac{d}{2}+1}\right)}$ sufficiently small, then inserting the above inequality into (4.11), we deduce

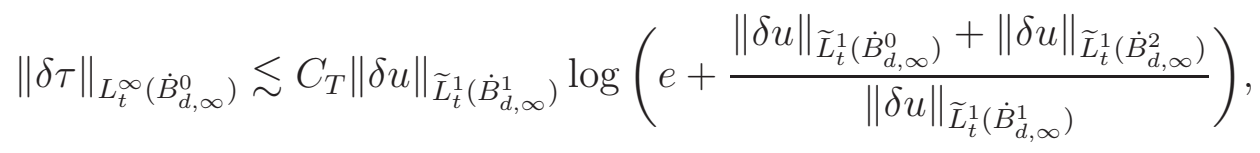

where $C_{T} \triangleq \exp \left(C\left\|u_{1}\right\|_{L_{T}^{1}\left(\dot{B}_{2,1}^{\frac{d}{2}+1}\right)}\right)\left(1+\left\|\tau_{1}\right\|_{L_{T}^{\infty}\left(\dot{B}_{2,1}^{\frac{d}{2}}\right)}+\left\|\tau_{2}\right\|_{L_{T}^{\infty}\left(\dot{B}_{2,1}^{\frac{d}{2}}\right)}\right)<\infty$.

For the estimate of $\delta u$. By Proposition 2.9, we get

$$
\|\delta u\|_{L_{t}^{\infty}\left(\dot{B}_{d, \infty}^{-1}\right)}+\|\delta u\|_{\widetilde{L}_{t}^{1}\left(\dot{B}_{d, \infty}^{1}\right)} \lesssim\|E\|_{\widetilde{L}_{t}^{1}\left(\dot{B}_{d, \infty}^{-1}\right)},
$$

where $E=\mu_{1} \nabla \cdot \delta \tau-u_{1} \cdot \nabla \delta u-\delta u \cdot \nabla u_{2}$. It follows from (3.37), Remark 2.7 and Proposition 2.5 that

$$
\begin{aligned}
\|E\|_{\widetilde{L}_{t}^{1}\left(\dot{B}_{d, \infty}^{-1}\right)} & \lesssim\|\delta \tau\|_{L_{t}^{1}\left(\dot{B}_{d, \infty}^{0}\right)}+\left\|u_{1} \otimes \delta u\right\|_{\widetilde{L}_{t}^{1}\left(\dot{B}_{d, \infty}^{0}\right)}+\left\|\delta u \otimes u_{2}\right\|_{\widetilde{L}_{t}^{1}\left(\dot{B}_{d, \infty}^{0}\right)} \\
& \lesssim\|\delta \tau\|_{L_{t}^{1}\left(\dot{B}_{d, \infty}^{0}\right)}+\left(\left\|u_{1}\right\|_{\widetilde{L}_{t}^{2}\left(\dot{B}_{d, 1}^{1}\right)}+\left\|u_{2}\right\|_{\widetilde{L}_{t}^{2}\left(\dot{B}_{d, 1}^{1}\right)}\right)\|\delta u\|_{\widetilde{L}_{t}^{2}\left(\dot{B}_{d, \infty}^{0}\right)} .
\end{aligned}
$$

Then in light of interpolation Theorem, we infer

$$
\begin{aligned}
& \|\delta u\|_{L_{t}^{\infty}\left(\dot{B}_{d, \infty}^{-1}\right)}+\|\delta u\|_{\widetilde{L}_{t}^{1}\left(\dot{B}_{d, \infty}^{1}\right)} \\
& \quad \lesssim\left(\left\|u_{1}\right\|_{\widetilde{L}_{t}^{2}\left(\dot{B}_{d, 1}^{1}\right)}+\left\|u_{2}\right\|_{\widetilde{L}_{t}^{2}\left(\dot{B}_{d, 1}^{1}\right)}\right)\left(\|\delta u\|_{L_{t}^{\infty}\left(\dot{B}_{d, \infty}^{-1}\right)}+\|\delta u\|_{\widetilde{L}_{t}^{1}\left(\dot{B}_{d, \infty}^{1}\right)}\right)+\|\delta \tau\|_{L_{t}^{1}\left(\dot{B}_{d, \infty}^{0}\right)} .
\end{aligned}
$$

Recall that

$$
u_{t}-\nu \Delta u=\mu_{1} \mathbb{P} \nabla \cdot \tau-\mathbb{P}(u \cdot \nabla u) .
$$

It is easy to verify that $\mu_{1} \mathbb{P} \nabla \cdot \tau-\mathbb{P}(u \cdot \nabla u) \in L_{T}^{1}\left(\dot{B}_{d, 1}^{0}\right)$ for the finite time $T$. Then from Proposition 2.9, we infer that $u_{i} \in \widetilde{L}_{T}^{\infty}\left(\dot{B}_{d, 1}^{0}\right) \cap \widetilde{L}_{T}^{1}\left(\dot{B}_{d, 1}^{2}\right)(i=1,2)$, therefore $u_{i} \in \widetilde{L}_{T}^{2}\left(\dot{B}_{d, 1}^{1}\right)$ by interpolation theorem. Taking $T$ small enough such that $\left\|u_{i}\right\|_{\widetilde{L}_{t}^{2} \dot{B}_{d, 1}^{1}}$ sufficiently small, then combining (4.12), (4.13), we have

$$
\begin{aligned}
\|\delta u\|_{L_{t}^{\infty}\left(\dot{B}_{d, \infty}^{-1}\right)}+ & \|\delta u\|_{\widetilde{L}_{t}^{1\left(\dot{B}_{d, \infty}^{1}\right)}} \\
& \lesssim C_{T} \int_{0}^{t}\|\delta u\|_{\widetilde{L}_{t^{\prime}\left(\dot{B}_{d, \infty}^{1}\right)}} \log \left(e+\frac{\left.\|\delta u\|_{\widetilde{L}_{t^{\prime}}^{1}\left(\dot{B}_{d, \infty}^{0}\right)}+\|\delta u\|_{\widetilde{L}_{t^{\prime}}^{1}\left(\dot{B}_{d, \infty}^{2}\right)}\right) \mathrm{d} t^{\prime} .}{\|\delta u\|_{\widetilde{L}_{t^{\prime}}^{1}\left(\dot{B}_{d, \infty}^{1}\right)}}\right.
\end{aligned}
$$

Denote $X(t) \triangleq\|\delta u\|_{L_{t}^{\infty}\left(\dot{B}_{d, \infty}^{-1}\right)}+\|\delta u\|_{\widetilde{L}_{t}^{1}\left(\dot{B}_{d, \infty}^{1}\right)}, V(t) \triangleq\|\delta u\|_{\widetilde{L}_{t}^{1}\left(\dot{B}_{d, \infty}^{0}\right)}+\|\delta u\|_{\widetilde{L}_{t}^{1}\left(\dot{B}_{d, \infty}^{2}\right)}$, we claim that $V(T)<\infty$. In fact

$$
\begin{aligned}
\|\delta u\|_{\widetilde{L}_{T}^{1}\left(\dot{B}_{d, \infty}^{0}\right)}+\|\delta u\|_{\widetilde{L}_{T}^{1}\left(\dot{B}_{d, \infty}^{2}\right)} & \lesssim \sum_{i=1}^{2} \int_{0}^{T}\left(\left\|u_{i}\right\|_{\dot{B}_{d, 1}^{0}}+\left\|u_{i}\right\|_{\dot{B}_{d, 1}^{2}}\right) \mathrm{d} t^{\prime} \\
& \lesssim \sum_{i=1}^{2}\left(T\left\|u_{i}\right\|_{L_{T}^{\infty} \dot{B}_{2,1}^{\frac{d}{2}-1}}+\left\|u_{i}\right\|_{L_{T}^{1} \dot{B}_{2,1}^{\frac{d}{2}+1}}\right) .
\end{aligned}
$$


We can rewrite (4.14) as follows

$$
X(t) \lesssim C_{T} \int_{0}^{t} X\left(t^{\prime}\right) \log \left(e+\frac{V(T)}{X\left(t^{\prime}\right)}\right) .
$$

As

$$
\int_{0}^{1} \frac{\mathrm{d} r}{r \log \left(e+\frac{V(T)}{r}\right)}=+\infty,
$$

Osgood lemma implies $X \equiv 0$ on $[0, T]$, whence also $\delta \tau \equiv 0$. Then a continuity argument ensures that $\left(u_{1}, \tau_{1}\right)=\left(u_{2}, \tau_{2}\right)$ on $\mathbb{R}^{+}$.

\section{ApPEndiX}

This section is devoted to the estimates of the convection terms which were used in Section 3.

First, let us give some definitions in paradifferential calculus in homogeneous spaces. We designate the homogeneous paraproduct of $v$ by $u$ as

$$
\dot{T}_{u} v \triangleq \sum_{q} \dot{S}_{q-1} \dot{\Delta}_{q} v
$$

and the homogeneous remainder of $u$ and $v$ as

$$
\dot{R}(u, v) \triangleq \sum_{q} \dot{\Delta}_{q} u \dot{\widetilde{\Delta}}_{q} v, \text { and } \quad \dot{\widetilde{\Delta}}_{q}=\dot{\Delta}_{q-1}+\dot{\Delta}_{q}+\dot{\Delta}_{q+1}
$$

Formally, we have the following homogeneous Bony decomposition:

$$
u v=\dot{T}_{u} v+\dot{T}_{v} u+\dot{R}(u, v) .
$$

The properties of continuity of homogeneous paraproduct and remainder on homogeneous hybrid Besov spaces are described as follows.

Proposition 5.1. For all $s_{1}, s_{2}, t_{1}, t_{2}$ such that $s_{1} \leq \frac{d}{2}$ and $s_{2} \leq \frac{d}{2}$, the following estimate holds

$$
\left\|T_{u} v\right\|_{\dot{B}^{s_{1}+t_{1}-\frac{d}{2}, s_{2}+t_{2}-\frac{d}{2}}} \lesssim\|u\|_{\dot{B}^{s_{1}, s_{2}}}\|v\|_{\dot{B}^{t_{1}, t_{2}}} .
$$

If $\min \left(s_{1}+t_{1}, s_{2}+t_{2}\right)>0$, then

$$
\|R(u, v)\|_{\dot{B}^{s_{1}+t_{1}-\frac{d}{2}, s_{2}+t_{2}-\frac{d}{2}}} \lesssim\|u\|_{\dot{B}^{s_{1}, s_{2}}}\|v\|_{\dot{B}^{t_{1}, t_{2}}} .
$$

If $u \in L^{\infty}$,

$$
\left\|T_{u} v\right\|_{\dot{B}^{t_{1}, t_{2}}} \lesssim\|u\|_{L^{\infty}}\|v\|_{\dot{B}^{t_{1}, t_{2}}}
$$

and, if $\min \left(t_{1}, t_{2}\right)>0$, then

$$
\|R(u, v)\|_{\dot{B}^{t_{1}, t_{2}}} \lesssim\|u\|_{L^{\infty}}\|v\|_{\dot{B}^{t_{1}, t_{2}}} .
$$

Remark 5.2. When $d \geq 2$, we have $\|u v\|_{\dot{B}^{\frac{d}{2}-1, \frac{d}{2}}} \lesssim\|u\|_{\dot{B}_{2,1}^{\frac{d}{2}}}\|v\|_{\dot{B}^{\frac{d}{2}-1, \frac{d}{2}}}$. 
Proposition 5.3. Let $u$ be a vector with $\nabla \cdot u=0$. Suppose that $-1-\frac{d}{2}<s_{1}, t_{1}, s_{2}, t_{2} \leq$ $1+\frac{d}{2}$. The following two estimates hold

$$
\begin{aligned}
\left|\left(\dot{\Delta}_{j}(u \cdot \nabla v), \dot{\Delta}_{j} v\right)\right| \lesssim & c_{j} 2^{-j \psi^{s_{1}, s_{2}}(j)}\|u\|_{\dot{B}_{2,1}^{\frac{d}{2}+1}}\|v\|_{\dot{B}^{s_{1}, s_{2}}}\left\|\dot{\Delta}_{j} v\right\|_{L^{2}}, \\
\mid\left(\dot{\Delta}_{j}(u \cdot \nabla v), \dot{\Delta}_{j} w\right)+ & \left(\dot{\Delta}_{j}(u \cdot \nabla w), \dot{\Delta}_{j} v\right) \mid \lesssim c_{j}\|u\|_{\dot{B}_{2,1}^{\frac{d}{2}+1}}\left(2^{-j \psi^{s_{1}, s_{2}}(j)}\|v\|_{\dot{B}^{s_{1}, s_{2}}}\left\|\dot{\Delta}_{j} w\right\|_{L^{2}}\right. \\
& \left.+2^{-j \psi^{t_{1}, t_{2}(j)}}\|w\|_{\dot{B}^{t_{1}, t_{2}}}\left\|\dot{\Delta}_{j} v\right\|_{L^{2}}\right) .
\end{aligned}
$$

where the function $\psi^{\alpha, \beta}(j)$ define as $\psi^{\alpha, \beta}(j)=\alpha$ if $j \leq 0, \psi^{\alpha, \beta}(j)=\beta$, if $j>0$, and $\sum_{j \in \mathbb{Z}} c_{j} \leq 1$.

One can refer to [1] for the proof of above two Propositions. Here we only have made a slight modification since the incompressible condition on $u$.

Next, we introduce a useful Proposition to deal with $[\mathbb{P} \operatorname{div}, u \cdot \nabla]$ type commutators.

Proposition 5.4. For any smooth tensor $\left[\tau^{i, j}\right]_{d \times d}$ and $d$ dimensional vector $u$, it always holds that

$$
\mathbb{P} \nabla \cdot(u \cdot \nabla \tau)=\mathbb{P}(u \cdot \nabla \mathbb{P} \nabla \cdot \tau)+\mathbb{P}(\nabla u \cdot \nabla \tau)-\mathbb{P}\left(\nabla u \cdot \nabla \Delta^{-1} \nabla \cdot \nabla \cdot \tau\right),
$$

where the ith component of $\nabla u \cdot \nabla \tau$ is

$$
[\nabla u \cdot \nabla \tau]^{i}=\sum_{j} \partial_{j} u \cdot \nabla \tau^{i, j}
$$

and also

$$
\left[\nabla u \cdot \nabla \Delta^{-1} \nabla \cdot \nabla \cdot \tau\right]^{i}=\partial_{i} u \cdot \nabla \Delta^{-1} \nabla \cdot \nabla \cdot \tau .
$$

For more detailed derivations, one can refer to the proof of the three dimensions in [24].

Acknowledgements. Q. Chen and X. Hao were supported by the National Natural Science Foundation of China (No.11671045).

\section{REFERENCES}

[1] H. Bahouri, J.Y. Chemin, R. Danchin, Fourier Analysis and Nonlinear Partial Differential Equations, Springer, Berlin/BerlinHeidelberg, (2011).

[2] R.B. Bird, C.F. Curtiss, R.C. Armstrong, O. Hassager, Dynamics of Polymetric Liquids, vol. 1, Fluid Mechanics, 2nd edn., Wiley, New York, (1987).

[3] M. Cannone, Ondelettes, paraproduits et Navier-Stokes, Nouveaux essais, Diderot éditeurs, Paris, (1995).

[4] M. Cannone, Harmonic analysis tools for solving the incompressible Navier-Stokes equations, Handbook of Mathematical fluid Dynamics, Vol. III, North-Holland, Amsterdam, (2004).

[5] M. Cannone, A generalization of a theorem by Kato on Naiver-Stokes equations, Revista Matemätica Iberoamericana, 13 (1997), 515-541.

[6] M. Cannone, Y. Meyer and F. Planchon, Solutions autosimilaires des équations de Navier-Stokes, Séminaire "Équations aux Dérivées Partielles" de l'École polytechnique, Exposé VIII, 1993-1994.

[7] J.-Y. Chemin, N. Masmoudi, About lifespan of regular solutions of equations related to viscoelastic fluids, SIAM J. Math. Anal., 33 (2001), 84-112.

[8] Q. Chen, C. Miao, Global well-posedness of viscoelastic fluids of Oldroyd type in Besov spaces, Nonlinear Anal., 68 (2008), 1928-1939. 
[9] Q. Chen, C. Miao, Z. Zhang, Global well-posedness for compressible Navier-Stokes equations with highly oscillating initial velocity, Comm. Pure Appl. Math., 63 (2010), 1173-1224.

[10] R. Danchin, Global existence in critical spaces for compressible Navier-Stokes equations, Invent. Math., 141 (2000), 579-614.

[11] R. Danchin, Global existence in critical sapaces for flows of compressible viscous and heat-conductive gases, Arch. Ration. Mech. Anal., 160 (2001), 1-39.

[12] R. Danchin, On the uniqueness in critical spaces for compresssible Navier-Stokes equatons, Nonlinear Differential Equations Appl., 12 (2005), 111-128.

[13] T.M. Elgindi, F. Rousset, Global regularity for some Oldroyd-B type models, Comm. Pure Appl. Math., 68 (2015), 2005-2021.

[14] T.M. Elgindi, J.L. Liu, Global wellposeness to the generalized Oldroyd type models in $\mathbb{R}^{3}$. J. Differential Equations, 259 (2015), 1958-1966.

[15] E. Fernandez-Cara, F. Guillén, R. R. Ortega, Existence et unicité de solution forte locale en temps pour des fluides non newtoniens de type Oldroyd (version $L^{s}-L^{r}$ ), C. R. Acad. Sci. Paris Sér. I Math., 319 (1994), 411-416.

[16] R. Zi, D. Fang, T. Zhang, Global solution to the incompressible Oldroyd-B type model in the critical $L^{p}$ framework: the case of the non-small coupling paramrter, Arch. Ration. Mech. Anal., 213 (2014), 651-687.

[17] D. Fang, R. Zi, Global solutions to the Oldroyd-B model with a class of large initial data, SIAM J.Math. Anal., 48 (2016), 1054-1084.

[18] H. Fujita, T. Kato, On the Navier-Stokes initial value problem I, Archive for Rational Mechanics and Analysis, 16 (1964), 269-315.

[19] C. Guillopé, J.-C. Saut, Existence results for the flow of viscoelastic fluids with a differential constitutive law, Nonlinear Anal., 15 (1990), 849-869.

[20] C. Guillopé, J.-C. Saut, Global existence and one-dimensional nonlinear stability of shearing motions of viscoelastic fluids of Oldroyd type, RAIRO Modél. Math. Anal.Numér., 24 (1990), 369-401.

[21] P.-L. Lions, N. Masmoudi, Global solutions for some Oldroyd models of non-Newtonian flows, Chin. Ann. Math. Ser. B, 21 (2000), 131-146.

[22] Z. Lei, N. Masmoudi, Y. Zhou, Remarks on the blowup criteria for Oldroyd models, J. Differential Equations, 248 (2010), 328-341.

[23] J.G. Oldroyd, Non-Newtonian effects in steady motion of some idealized elastico-viscous liquids, Proc. Roy. Soc. Edinburgh Sect. A, 245 (1958), 278-297.

[24] Y. Zhu, Global small solutions of $3 D$ incompressible Oldroyd-B model without damping mechanism, Journal of Functional Analysis, 274 (2017), 2039-2060.

Institute of Applied Physics and Computational Mathematics, Beijing 100088, China

E-mail address: chen_qionglei@iapcm.ac.cn

The Graduate School of China Academy of Engineering Physics, Beijing 100088, China E-mail address: xn_hao@163.com 